\title{
FRACTAL JUST INFINITE NIL LIE SUPERALGEBRA OF FINITE WIDTH
}

\author{
OTTO AUGUSTO DE MORAIS COSTA AND VICTOR PETROGRADSKY
}

\begin{abstract}
The Grigorchuk and Gupta-Sidki groups play fundamental role in modern group theory. They are natural examples of self-similar finitely generated periodic groups. As their natural analogues, there are constructions of nil Lie $p$-algebras over a field of characteristic 2 [40] and arbitrary positive characteristic [52]. In characteristic zero, similar examples of Lie algebras do not exist by a result of Martinez and Zelmanov 32.

The second author constructed analogues of the Grigorchuk and Gupta-Sidki groups in the world of Lie superalgebras of arbitrary characteristic, the virtue of that construction is that the Lie superalgebras have clear monomial bases [11. That Lie superalgebras have slow polynomial growth and are graded by multidegree in the generators. In particular, a self-similar Lie superalgebra $\mathbf{Q}$ is $\mathbb{Z}^{3}$-graded by multidegree in 3 generators, its $\mathbb{Z}^{3}$-components lie inside an elliptic paraboloid in space, the components are at most onedimensional, thus, the $\mathbb{Z}^{3}$-grading of $\mathbf{Q}$ is fine. An analogue of the periodicity is that homogeneous elements of the grading $\mathbf{Q}=\mathbf{Q}_{\overline{0}} \oplus \mathbf{Q}_{\overline{1}}$ are ad-nilpotent. In particular, $\mathbf{Q}$ is a nil finely graded Lie superalgebra, which shows that an extension of the mentioned result of Martinez and Zelmanov 32 to the Lie superalgebras of characteristic zero is not valid. But computations with $\mathbf{Q}$ are rather technical.

In this paper, we construct a similar but simpler and "smaller" example. Namely, we construct a 2generated fractal Lie superalgebra $\mathbf{R}$ over arbitrary field. We find a clear monomial basis of $\mathbf{R}$ and, unlike many examples studied before, we find also a clear monomial basis of its associative hull $\mathbf{A}$, the latter has a quadratic growth. The algebras $\mathbf{R}$ and $\mathbf{A}$ are $\mathbb{Z}^{2}$-graded by multidegree in the generators, positions of their $\mathbb{Z}^{2}$-components are bounded by pairs of logarithmic curves on plane. The $\mathbb{Z}^{2}$-components of $\mathbf{R}$ are at most one-dimensional, thus, the $\mathbb{Z}^{2}$-grading of $\mathbf{R}$ is fine. As an analogue of periodicity, we establish that homogeneous elements of the grading $\mathbf{R}=\mathbf{R}_{\overline{0}} \oplus \mathbf{R}_{\overline{1}}$ are ad-nilpotent. In case of $\mathbb{N}$-graded algebras, a close analogue to being simple is being just infinite. Unlike previous examples of Lie superalgebras, we are able to prove that $\mathbf{R}$ is just infinite, but not hereditary just infinite. Our example is close to the smallest possible example, because $\mathbf{R}$ has a linear growth with a growth function $\gamma_{\mathbf{R}}(m) \approx 3 m$, as $m \rightarrow \infty$. Moreover, $\mathbf{R}$ is of finite width 4 (char $K \neq 2$ ). In case char $K=2$, we obtain a Lie algebra of width 2 that is not thin.

Thus, we have got a more handy analogue of the Grigorchuk and Gupta-Sidki groups. The constructed Lie superalgebra $\mathbf{R}$ is of linear growth, of finite width 4 , and just infinite. It also shows that an extension of the result of Martinez and Zelmanov [32] to the Lie superalgebras of characteristic zero is not valid.
\end{abstract}

\section{Introduction: Self-Similar groups AND algebras}

1.1. Golod-Shafarevich algebras and groups. The General Burnside Problem puts the question whether a finitely generated periodic group is finite. The first negative answer was given by Golod and Shafarevich, who proved that, for each prime $p$, there exists a finitely generated infinite $p$-group [17, 18. The construction is based on a famous construction of a family of finitely generated infinite dimensional associative nilalgebras [17. This construction also yields examples of infinite dimensional finitely generated Lie algebras $L$ such that $(\operatorname{ad} x)^{n(x, y)}(y)=0$, for all $x, y \in L$, the field being arbitrary [19]. The field being of positive characteristic $p$, one obtains an infinite dimensional finitely generated restricted Lie algebra $L$ such that the $p$-mapping is nil, namely, $x^{\left[p^{n(x)}\right]}=0$, for all $x \in L$. This gives a negative answer to a question of Jacobson whether a finitely generated restricted Lie algebra $L$ is finite dimensional provided that each element $x \in L$ is algebraic, i.e. satisfies some $p$-polynomial $f_{p, x}(x)=0([26$, Ch. 5 , ex. 17]). It is known that the construction of Golod yields associative nil-algebras of exponential growth. Using specially chosen relations, Lenagan and Smoktunowicz constructed associative nil-algebras of polynomial growth 31. On further developments concerning Golod-Shafarevich algebras and groups see 62, 14.

A close by spirit but different construction was motivated by respective group-theoretic results. A restricted Lie algebra $G$ is called large if there is a subalgebra $H \subset G$ of finite codimension such that $H$ admits a surjective homomorphism on a nonabelian free restricted Lie algebra. Let $K$ be a perfect at most countable field of positive characteristic. Then there exist infinite dimensional finitely generated nil restricted Lie algebras over $K$ that are residually finite dimensional and direct limits of large restricted Lie algebras [3].

2000 Mathematics Subject Classification. 16P90, 16N40, 16S32, 17B50, 17B65, 17B66, $17 \mathrm{~B} 70$.

Key words and phrases. Lie superalgebras, restricted Lie algebras, growth, linear growth, Gelfand-Kirillov dimension, selfsimilar algebras, fractal algebras, nil-algebras, graded algebras, just infinite, finite width, thin Lie algebras, filiform Lie algebras, Lie algebras of differential operators.

The second author was partially supported by grants CNPq 309542/2016-2, FAPESP 2016/18068-9 . 
1.2. Grigorchuk and Gupta-Sidki groups. The construction of Golod is rather undirect, Grigorchuk gave a direct and elegant construction of an infinite 2-group generated by three elements of order 2 [20]. This group was defined as a group of transformations of the interval $[0,1]$ from which rational points of the form $\left\{k / 2^{n} \mid 0 \leq k \leq 2^{n}, n \geq 0\right\}$ are removed. For each prime $p \geq 3$, Gupta and Sidki gave a direct construction of an infinite $p$-group on two generators, each of order $p$ [23. This group was constructed as a subgroup of an automorphism group of an infinite regular tree of degree $p$.

The Grigorchuk and Gupta-Sidki groups are counterexamples to the General Burnside Problem. Moreover, they gave answers to important problems in group theory. So, the Grigorchuk group and its further generalizations are first examples of groups of intermediate growth 21, thus answering in negative to a conjecture of Milnor that groups of intermediate growth do not exist. The construction of Gupta-Sidki also yields groups of subexponential growth [15. The Grigorchuk and Gupta-Sidki groups are self-similar. Now self-similar, and so called branch groups, form a well-established area in group theory, see for further developments [22, 36. Below we discuss existence of analogues of the Grigorchuk and Gupta-Sidki groups for other algebraic structures.

1.3. Self-similar nil graded associative algebras. The study of these groups lead to investigation of group rings and other related associative algebras [57. In particular, there appeared self-similar associative algebras defined by matrices in a recurrent way [5]. Sidki suggested two examples of self-similar associative matrix algebras [56]. A more general family of self-similar associative algebras was introduced in [45, this family generalizes the second example of Sidki [56], also it yields a realization of a Fibonacci restricted Lie algebras (see below) in terms of self-similar matrices 45. Another important feature of some associative algebras $A$ constructed in [45] is that they are sums of two locally nilpotent subalgebras $A=A_{+} \oplus A_{-}$(see similar decompositions (11) below). Recall that an algebra is said locally nilpotent if every finitely generated subalgebra is nilpotent. But the desired analogues of the Grigorchuk and Gupta-Sidki groups should be associative self-similar nil-algebras, in a standard way yielding new examples of finitely generated periodic groups. But such examples are not known yet. On similar open problems in theory of infinite dimensional algebras see review [63].

1.4. Self-similar nil restricted Lie algebras, Fibonacci Lie algebra. Unlike associative algebras, for restricted Lie algebras, there are known natural analogues of the Grigorchuk and Gupta-Sidki groups. Namely, over a field of characteristic 2, the second author constructed an example of an infinite dimensional restricted Lie algebra $\mathbf{L}$ generated by two elements, called a Fibonacci restricted Lie algebra [40. Let char $K=p=2$ and $R=K\left[t_{i} \mid i \geq 0\right] /\left(t_{i}^{p} \mid i \geq 0\right)$ a truncated polynomial ring. Put $\partial_{i}=\frac{\partial}{\partial t_{i}}, i \geq 0$. Define the following two derivations of $R$ :

$$
\begin{array}{ll}
v_{1}=\partial_{1}+t_{0}\left(\partial_{2}+t_{1}\left(\partial_{3}+t_{2}\left(\partial_{4}+t_{3}\left(\partial_{5}+t_{4}\left(\partial_{6}+\cdots\right)\right)\right)\right)\right) \\
v_{2}= & \partial_{2}+t_{1}\left(\partial_{3}+t_{2}\left(\partial_{4}+t_{3}\left(\partial_{5}+t_{4}\left(\partial_{6}+\cdots\right)\right)\right)\right)
\end{array}
$$

These two derivations generate a restricted Lie algebra $\mathbf{L}=\operatorname{Lie}_{p}\left(v_{1}, v_{2}\right) \subset$ Der $R$ and an associative algebra $\mathbf{A}=\operatorname{Alg}\left(v_{1}, v_{2}\right) \subset$ End $R$. By Bergman's theorem, the Gelfand-Kirillov dimension of an associative algebra cannot belong to the interval $(1,2) 29$. Such a gap for Lie algebras does not exist, the Gelfand-Kirillov dimension of a finitely generated Lie algebra can be arbitrary number $\{0\} \cup[1,+\infty)$ 39]. The Fibonacci Lie algebra has slow polynomial growth with Gelfand-Kirillov dimension GKdim $\mathbf{L}=\log _{(\sqrt{5}+1) / 2} 2 \approx 1.44[40$ ]. Further properties of the Fibonacci restricted Lie algebra and its generalizations are studied in [44, 46].

Probably, the most interesting property of $\mathbf{L}$ is that it has a nil $p$-mapping [40, which is an analog of the periodicity of the Grigorchuk and Gupta-Sidki groups. We do not know whether the associative hull $\mathbf{A}$ is a nil-algebra. We have a weaker statement. The algebras $\mathbf{L}, \mathbf{A}$, and the augmentation ideal of the restricted enveloping algebra $\mathbf{u}=\omega u(\mathbf{L})$ are direct sums of two locally nilpotent subalgebras [4]:

$$
\mathbf{L}=\mathbf{L}_{+} \oplus \mathbf{L}_{-}, \quad \mathbf{A}=\mathbf{A}_{+} \oplus \mathbf{A}_{-}, \quad \mathbf{u}=\mathbf{u}_{+} \oplus \mathbf{u}_{-} .
$$

There are examples of infinite dimensional associative algebras which are direct sums of two locally nilpotent subalgebras [28, 12. Infinite dimensional restricted Lie algebras can have different decompositions into a direct sum of two locally nilpotent subalgebras [48.

In case of arbitrary prime characteristic, Shestakov and Zelmanov suggested an example of a finitely generated restricted Lie algebra with a nil $p$-mapping [52. That example yields the same decompositions (10) for some primes [30, 45. An example of a $p$-generated nil restricted Lie algebra $L$, characteristic $p$ being arbitrary, was studied in [48. The virtue of that example is that for all primes $p$ we have the same decompositions (11) into direct sums of two locally nilpotent subalgebras. But computations for that example are rather complicated. 
Observe that only the original example has a clear monomial basis [40, 44]. In other examples, elements of a Lie algebra are linear combinations of monomials, to work with such linear combinations is sometimes an essential technical difficulty, see e.g. [52, 48]. A family of nil restricted Lie algebras of slow polynomial growth having clear monomial bases is constructed in [42], these algebras are close relatives of a two-generated Lie superalgebra of [4]. As a particular case, we obtain a continuum subfamily of non-isomorphic nil restricted Lie algebras with the Gelfand-Kirillov dimension equal to one, but their growth is not linear [41.

1.5. Narrow groups and Lie algebras. Let $G$ be a group and $G=G_{1} \supseteq G_{2} \supseteq \cdots$ its lower central series. One constructs a related $\mathbb{N}$-graded Lie algebra $L_{K}(G)=\oplus_{i \geq 1} L_{i}$, where $L_{i}=G_{i} / G_{i+1} \otimes_{\mathbb{Z}} K, i \geq 1$. A product is given by $\left[a_{i} G_{i+1}, b_{j} G_{j+1}\right]=\left(a_{i}, b_{j}\right) G_{i+j+1}$, where $\left(a_{i}, b_{j}\right)=a_{i}^{-1} b_{j}^{-1} a_{i} b_{j}$ is the group commutator.

A residually $p$-group $G$ is said of finite width if all factors $G_{i} / G_{i+1}$ are finite groups with uniformly bounded orders. The Grigorchuk group $G$ is of finite width, namely, $\operatorname{dim}_{\mathbb{F}_{2}} G_{i} / G_{i+1} \in\{1,2\}$ for $i \geq 2$ [51, 7 . In particular, the respective Lie algebra $L=L_{K}(G)=\oplus_{i>1} L_{i}$ has a linear growth. Bartholdi presented $L_{K}(G)$ as a self-similar restricted Lie algebra and proved that the restricted Lie algebra $L_{\mathbb{F}_{2}}(G)$ is nil while $L_{\mathbb{F}_{4}}(G)$ is not nil [6]. Also, $L_{K}(G)$ is nil graded, namely, for any homogeneous element $x \in L_{i}, i \geq 1$, the mapping ad $x$ is nilpotent, because $G$ is periodic.

Shalev and Zelmanov suggested that narrowness conditions for Lie algebras deserve systematic study because the most important algebras are narrow in some sense [54, 55]. Infinite dimensional $\mathbb{N}$-graded Lie algebras $L=\bigoplus_{n=1}^{\infty} L_{n}$ with one-dimensional components in characteristic zero were classified by Fialowski [16]. A Lie algebra $L$ is called of maximal class (or filiform), if the associated graded algebra with respect to the lower central series gr $L=\bigoplus_{n=1}^{\infty} \operatorname{gr} L_{n}$, where gr $L_{n}=L^{n} / L^{n+1}, n \geq 1$, satisfies

$$
\operatorname{dim} \operatorname{gr} L_{1}=2, \quad \operatorname{dim} \operatorname{gr} L_{n} \leq 1, n \geq 2, \quad \operatorname{gr} L_{n+1}=\left[\operatorname{gr} L_{1}, \operatorname{gr} L_{n}\right], n \geq 1,
$$

in particular, gr $L$ is generated by gr $L_{1}$. It follows from the paper of Vergne 61 that in zero characteristic there exists a unique infinite dimensional $\mathbb{N}$-graded Lie algebra whose grading satisfies (2). In zero characteristic their exist uncountably many pairwise non-isomorphic $\mathbb{N}$-graded filiform Lie algebras of width one in dimensions 7-11 34 .

An infinite dimensional filiform Lie algebra $L$ has the smallest nontrivial growth function: $\gamma_{L}(n)=n+1$, $n \geq 1$. In case of positive characteristic, there are uncountably many such algebras [10]. Nevertheless, in case $p>2$, they were classified in [11. There are generalizations of filiform Lie algebras. A graded Lie algebra $L=\bigoplus_{n=1}^{\infty} L_{n}$ is said thin if $\operatorname{dim} L_{1}=2$ and it satisfies the covering property: $L_{n+1}=\left[L_{1}, z\right]$ for any $0 \neq z \in L_{n}$ and all $n \geq 1$ [9]. In particular, $\operatorname{dim} L_{n} \leq 2$ for all $n \geq 1$. Components of dimension 2 are called diamonds. This notion appeared in a theory of similarly defined thin groups [9].

Naturally $\mathbb{N}$-graded Lie algebras over $\mathbb{R}$ and $\mathbb{C}$ satisfying the condition $\operatorname{dim} L_{n}+\operatorname{dim} L_{n+1} \leq 3, n \geq 1$, are classified recently by Millionschikov [35. More generally, an $\mathbb{N}$-graded Lie algebra $L=\bigoplus_{n=1}^{\infty} L_{n}$ is said of finite width $d$ in the case that $\operatorname{dim} L_{n} \leq d, n \geq 1$, the integer $d$ being minimal.

Pro-p-groups and $\mathbb{N}$-graded Lie algebras cannot be simple. Instead one has an important notion of being just infinite, namely, not having non-trivial normal subgroups (ideals) of infinite index (codimension). A group (algebra) is said hereditary just infinite if and only if any normal subgroup (ideal) of finite index (codimension) is just infinite. The Gupta-Sidki groups were the first in the class of periodic groups to be shown to be just infinite [24]. The Grigorchuk group is also just infinite but not hereditary just infinite [22].

1.6. Lie algebras over a field of characteristic zero. Since the Grigorchuk group is periodic and of finite width, its appropriate analogue should be a "small" ad-nil Lie algebra. For example, a Lie algebra should be of finite width, implying a linear growth. In the next result, a weaker assumption that components of a grading are bounded implies that the growth of finitely generated subalgebras is polynomial. Informally speaking, there are no "natural analogues" of the Grigorchuk and Gupta-Sidki groups in the world of Lie algebras of characteristic zero in terms of the following result sensu stricto.

Theorem 1.1 (Martinez and Zelmanov 32]). Let $L=\oplus_{\alpha \in \Gamma} L_{\alpha}$ be a Lie algebra over a field $K$ of characteristic zero graded by an abelian group $\Gamma$. Suppose that

i) there exists $d>0$ such that $\operatorname{dim}_{K} L_{\alpha} \leq d$ for all $\alpha \in \Gamma$,

ii) every homogeneous element $a \in L_{\alpha}, \alpha \in \Gamma$, is ad-nilpotent.

Then the Lie algebra $L$ is locally nilpotent. 
1.7. Fractal nil graded Lie superalgebras $\mathbf{R}$ and $\mathbf{Q}$. In the world of Lie superalgebras of an arbitrary characteristic, the second author constructed analogues of the Grigorchuk and Gupta-Sidki groups [41. Namely, two Lie superalgebras $\mathbf{R}, \mathbf{Q}$ were constructed, which are also analogues of the Fibonacci restricted Lie algebra and other restricted Lie algebras mentioned above. The constructions of both Lie superalgebras $\mathbf{R}, \mathbf{Q}$ are similar, computations for $\mathbf{R}$ are simpler, but $\mathbf{Q}$ enjoys some more specific interesting properties. The virtue of both examples is that they have clear monomial bases. They have slow polynomial growth, namely, GKdim $\mathbf{R}=\log _{3} 4 \approx 1.26$ and GKdim $\mathbf{Q}=\log _{3} 8 \approx 1.89$. Thus, both Lie superalgebras are of infinite width. In both examples, ad $a$ is nilpotent, $a$ being an even or odd element with respect to $\mathbb{Z}_{2}$-gradings as Lie superalgebras. This property is an analogue of the periodicity of the Grigorchuk and Gupta-Sidki groups. The Lie superalgebra $\mathbf{R}$ is $\mathbb{Z}^{2}$-graded, while $\mathbf{Q}$ has a natural fine $\mathbb{Z}^{3}$-gradation with at most one-dimensional components (See on importance of fine gradins for Lie and associative algebras [4, 13. There are examples of simple $\mathbb{Z}^{2}$-graded Lie algebras in characteristic zero with one-dimensional components [25]). In particular, $\mathbf{Q}$ is a nil finely graded Lie superalgebra, which shows that an extension of Theorem 1.1 (Martinez and Zelmanov [32]) for the Lie superalgebras of characteristic zero is not valid. Also, $\mathbf{Q}$ has a $\mathbb{Z}^{2}$-gradation which yields a continuum of different decompositions into sums of two locally nilpotent subalgebras $\mathbf{Q}=\mathbf{Q}_{+} \oplus \mathbf{Q}_{-}$. Both Lie superalgebras are self-similar. They also contain infinitely many copies of themselves, we call them fractal due to the last property.

In Section 2 we supply basic definitions. In Section 3 we give a construction of our Lie superalgebra $\mathbf{R}$ (this notation was also used for the 2-generated Lie superalgebra $\mathbf{R}$ of [41], which shall be mentioned in the present paper one more time) and formulate its basic properties.

\section{Basic definitions: (Restricted) Lie superalgebras, Growth}

Superalgebras appear naturally in physics and mathematics [27, 53, 2. By $K$ denote the ground field, $\langle S\rangle_{K}$ a linear span of a subset $S$ in a $K$-vector space. Put $\mathbb{Z}_{2}=\{\overline{0}, \overline{1}\}$, the group of order 2. A superalgebra $A$ is a $\mathbb{Z}_{2}$-graded algebra $A=A_{\overline{0}} \oplus A_{\overline{1}}$. The elements $a \in A_{\alpha}$ are called homogeneous of degree $\operatorname{deg} a=\alpha$, $\alpha \in \mathbb{Z}_{2}$. The elements of $A_{\overline{0}}$ are even, those of $A_{\overline{1}}$ odd. In what follows, if $\operatorname{deg} a$ enters an expression, then it is assumed that $a$ is homogeneous of degree $\operatorname{deg} a \in \mathbb{Z}_{2}$, and the expression extends to the other elements by linearity. Let $A, B$ be superalgebras, a tensor product $A \otimes B$ is the superalgebra whose space is the tensor product of the spaces $A$ and $B$ with the induced $\mathbb{Z}_{2}$-grading and the product:

$$
\left(a_{1} \otimes b_{1}\right)\left(a_{2} \otimes b_{2}\right)=(-1)^{\operatorname{deg} b_{1} \cdot \operatorname{deg} a_{2}} a_{1} a_{2} \otimes b_{1} b_{2}, \quad a_{i} \in A, b_{i} \in B .
$$

An associative superalgebra $A$ is a $\mathbb{Z}_{2}$-graded associative algebra $A=A_{\overline{0}} \oplus A_{\overline{1}}$. A Lie superalgebra is a $\mathbb{Z}_{2}$-graded algebra $L=L_{\overline{0}} \oplus L_{\overline{1}}$ with an operation [ , ] satisfying the axioms:

- $[x, y]=-(-1)^{\operatorname{deg} x \cdot \operatorname{deg} y}[y, x], \quad$ (super-anticommutativity);

- $[x,[y, z]]=[[x, y], z]+(-1)^{\operatorname{deg} x \cdot \operatorname{deg} y}[y,[x, z]], \quad(J a c o b i$ identity $)$.

All commutators in the present paper are supercommutators. Long commutators are right-normed: $[x, y, z]=$ $[x,[y, z]]$. We use a standard notation ad $x(y)=[x, y]$, where $x, y \in L$.

Assume that $A=A_{\overline{0}} \oplus A_{\overline{1}}$ is an associative superalgebra. One obtains a Lie superalgebra $A^{(-)}$by supplying the same vector space $A$ with a supercommutator:

$$
[x, y]=x y-(-1)^{\operatorname{deg} x \cdot \operatorname{deg} y} y x, \quad x, y \in A .
$$

If $A^{(-)}$is abelian, then $A$ is called supercommutative. Let $L$ be a Lie superalgebra, one defines a universal enveloping algebra $U(L)=T(L) /\left(x \otimes y-(-1)^{\operatorname{deg} x \cdot \operatorname{deg} y} y \otimes x-[x, y] \mid x, y \in L\right)$, where $T(L)$ is the tensor algebra of the vector space $L$. Now, the product in $L$ coincides with the supercommutator in $U(L)^{(-)}$. A basis of $U(L)$ is given by PBW-theorem [2, 53.

In case char $K=2,3$ the axioms of the Lie superalgebra have to be augmented ([2, section 1.10], 8], [41]).

- $(\operatorname{char} K=3)[z,[z, z]]=0, z \in L_{\overline{1}}$.

Substituting $x=y \in L_{\overline{1}}$ in the Jacobi identity, we get $2(\operatorname{ad} x)^{2} z=[[x, x], z]$. In case char $K \neq 2$ we get an identity

$$
(\operatorname{ad} x)^{2} z=\frac{1}{2}[[x, x], z], \quad x \in L_{\overline{1}}, z \in L .
$$

In the present paper we study Lie superalgebras of the form $A^{(-)}$, they have squares: $[x, x]=2 x^{2}, x \in A_{\overline{1}}^{(-)}$. One obtains an identity that is also valid for char $K=2$ :

$$
(\operatorname{ad} x)^{2} z=\left[x^{2}, z\right], \quad x \in A_{\overline{1}}^{(-)}, z \in A^{(-)} .
$$

In case char $K=2$, we add more axioms for the Lie superalgebras: 
- there exists a quadratic mapping (a formal square): ( ) ${ }^{[2]}: L_{\overline{1}} \rightarrow L_{\overline{0}}, x \mapsto x^{[2]}, x \in L_{\overline{1}}$, satisfying:

$$
\begin{aligned}
(\lambda x)^{[2]} & =\lambda^{2} x^{[2]}, \quad x \in L_{\overline{1}}, \quad \lambda \in K ; \\
(x+y)^{[2]} & =x^{[2]}+[x, y]+y^{[2]} ; \quad x, y \in L_{\overline{1}} ; \\
(\operatorname{ad} x)^{2} z & =\left[x^{[2]}, z\right], \quad x \in L_{\overline{1}}, \quad z \in L, \quad \text { (a formal substitute of (33)); }
\end{aligned}
$$

- $[x, x]=0, x \in L_{\overline{0}}$. By putting $y=x$ in the second relation above, we get $[y, y]=0, y \in L_{\overline{1}}$.

In other words, a Lie superalgebra in case char $K=2$ is just a $\mathbb{Z}_{2}$-graded Lie algebra supplied with a quadratic mapping $L_{\overline{1}} \rightarrow L_{\overline{0}}$, which is similar to the $p$-mapping (see below). In case $p=2$, to get the universal enveloping algebra, we additionally factor out $\left\{y \otimes y-y^{[2]} \mid y \in L_{\overline{1}}\right\}$.

We shall consider also Lie super-rings. These are $\mathbb{Z}_{2}$-graded $\mathbb{Z}$-algebras whose product satisfies the superanticommutativity and the Jacobi identity. Since Lie algebras over a field of char $K=2$ should be considered as such rings, we also assume existence of the formal quadratic mapping satisfying the axioms above.

In all cases, the quadratic mapping will be denoted by $x^{2}, x \in L_{\overline{1}}$, it also coincides with the ordinary square in the universal (or restricted) enveloping algebra $U(L)$.

Let $V=V_{\overline{0}} \oplus V_{\overline{1}}$ be a vector space, we say that it is $\mathbb{Z}_{2}$-graded. The associative algebra of all endomorphisms of the space End $V$ is an associative superalgebra: End $V=\operatorname{End}_{\overline{0}} V \oplus \operatorname{End}_{\overline{1}} V$, where $\operatorname{End}_{\alpha} V=\{\phi \in$ End $\left.V \mid \phi\left(V_{\beta}\right) \subset V_{\alpha+\beta}, \beta \in \mathbb{Z}_{2}\right\}, \alpha \in \mathbb{Z}_{2}$. Thus, End ${ }^{(-)} V$ is a Lie superalgebra, called the general linear superalgebra $g l(V)$.

Let $A=A_{\overline{0}} \oplus A_{\overline{1}}$ be a $\mathbb{Z}_{2}$-graded algebra of arbitrary signature. A linear mapping $\phi \in \operatorname{End}_{\beta} A, \beta \in \mathbb{Z}_{2}$, is a superderivative of degree $\beta$ if it satisfies

$$
\phi(a \cdot b)=\phi(a) \cdot b+(-1)^{\beta \cdot \operatorname{deg}(a)} a \cdot \phi(b), \quad a, b \in A .
$$

Denote by $\operatorname{Der}_{\alpha} A \subset \operatorname{End}_{\alpha} A$ the space of all superderivatives of degree $\alpha \in \mathbb{Z}_{2}$. One checks that Der $A=$ $\operatorname{Der}_{\overline{0}} A \oplus \operatorname{Der}_{\overline{1}} A$ is a subalgebra of the Lie superalgebra End ${ }^{(-)} A$. In this paper by a derivation we always mean a superderivation.

Let $L$ be a Lie algebra over a field $K$ of characteristic $p>0$. Then $L$ is called a restricted Lie algebra (or Lie $p$-algebra), if it is additionally supplied with a unary operation $x \mapsto x^{[p]}, x \in L$, that satisfies the following axioms [26, 59, 60]:

- $(\lambda x)^{[p]}=\lambda^{p} x^{[p]}$, for $\lambda \in K, x \in L$;

- $\operatorname{ad}\left(x^{[p]}\right)=(\operatorname{ad} x)^{p}, x \in L$

- $(x+y)^{[p]}=x^{[p]}+y^{[p]}+\sum_{i=1}^{p-1} s_{i}(x, y), x, y \in L$, where $i s_{i}(x, y)$ is the coefficient of $t^{i-1}$ in the polynomial $\operatorname{ad}(t x+y)^{p-1}(x) \in L[t]$.

This notion is motivated by the following observation. Let $A$ be an associative algebra over a field $K$, char $K=p>0$. Then the mapping $x \mapsto x^{p}, x \in A^{(-)}$, satisfies these conditions considered in the Lie algebra $A^{(-)}$.

A restricted Lie superalgebra $L=L_{\overline{0}} \oplus L_{\overline{1}}$ is a Lie superalgebra such that the even component $L_{\overline{0}}$ is a restricted Lie algebra and $L_{\overline{0}}$-module $L_{\overline{1}}$ is restricted, i.e. $\operatorname{ad}\left(x^{[p]}\right) y=(\operatorname{ad} x)^{p} y$, for all $x \in L_{\overline{0}}, y \in L_{\overline{1}}(\operatorname{see}$. e.g. 33, 2, 38, ). Remark that in case char $K=2$, the restricted Lie superalgebras and $\mathbb{Z}_{2}$-graded restricted Lie algebras are the same objects. (Let $L=L_{\overline{0}} \oplus L_{\overline{1}}$ be a restricted Lie superalgebra, it has the $p$-mapping on the even part: $L_{\overline{0}} \rightarrow L_{\overline{0}}$ and the formal square on the odd part: $L_{\overline{1}} \rightarrow L_{\overline{0}}$. We obtain a $p$-mapping on the whole of algebra by setting $\left.(x+y)^{[2]}=x^{[2]}+y^{[2]}+[x, y], x \in L_{\overline{0}}, y \in L_{\overline{1}}\right)$.

Let $L$ be a restricted Lie (super)algebra, and $J$ the ideal of the universal enveloping algebra $U(L)$ generated by all elements $x^{[p]}-x^{p}, x \in L_{\overline{0}}$. Then $u(L)=U(L) / J$ is the restricted enveloping algebra of $L$. In this algebra, the formal operation $x^{[p]}$ coincides with the ordinary power $x^{p}$ for all $x \in L_{\overline{0}}$. One has an analogue of PBW-theorem describing a basis of $u(L)$ [26, p. 213], 2].

Let $L$ be a Lie (super)algebra. One defines the lower central series as $L^{1}=L$ and $L^{n}=\left[L, L^{n-1}\right], n \geq 2$. In case char $K=2$ the space above is augmented by $\left\langle x^{2} \mid x \in\left(L^{[n / 2]}\right)_{\overline{1}}\right\rangle_{K}$. In case of a restricted Lie (super)algebra, we also add $\left\langle x^{p} \mid x \in\left(L^{[n / p]}\right)_{\overline{0}}\right\rangle_{K}$.

We recall the notion of growth. Let $A$ be an associative (or Lie) (super)algebra generated by a finite set $X$. Denote by $A^{(X, n)}$ the subspace of $A$ spanned by all monomials in $X$ of length not exceeding $n, n \geq 0$. In case of a Lie superalgebra of char $K=2$ we also consider formal squares of odd monomials of length at most $n / 2$. If $A$ is a restricted Lie algebra, put $A^{(X, n)}=\left\langle\left[x_{1}, \ldots, x_{s}\right]^{p^{k}} \mid x_{i} \in X, s p^{k} \leq n\right\rangle_{K}$ [37]. Similarly, one defines the growth for restricted Lie superalgebras. In either situation, one defines an (ordinary) growth function:

$$
\gamma_{A}(n)=\gamma_{A}(X, n)=\operatorname{dim}_{K} A^{(X, n)}, \quad n \geq 0
$$


Let $f, g: \mathbb{N} \rightarrow \mathbb{R}^{+}$be eventually increasing and positive valued functions. Write $f(n) \preccurlyeq g(n)$ if and only if there exist positive constants $N, C$ such that $f(n) \leq g(C n)$ for all $n \geq N$. Introduce equivalence $f(n) \sim g(n)$ if and only if $f(n) \preccurlyeq g(n)$ and $g(n) \preccurlyeq f(n)$. Different generating sets of an algebra yield equivalent growth functions 29 .

It is well known that the exponential growth is the highest possible growth for finitely generated Lie and associative algebras. A growth function $\gamma_{A}(n)$ is compared with polynomial functions $n^{\alpha}, \alpha \in \mathbb{R}^{+}$, by computing the upper and lower Gelfand-Kirillov dimensions [29]:

$$
\begin{aligned}
& \operatorname{GKdim} A=\varlimsup_{n \rightarrow \infty} \frac{\ln \gamma_{A}(n)}{\ln n}=\inf \left\{\alpha>0 \mid \gamma_{A}(n) \preccurlyeq n^{\alpha}\right\} ; \\
& \underline{\operatorname{GKdim}} A=\varliminf_{n \rightarrow \infty} \frac{\ln \gamma_{A}(n)}{\ln n}=\sup \left\{\alpha>0 \mid \gamma_{A}(n) \succcurlyeq n^{\alpha}\right\} .
\end{aligned}
$$

Assume that generators $X=\left\{x_{1}, \ldots, x_{k}\right\}$ are assigned positive weights $\operatorname{wt}\left(x_{i}\right)=\lambda_{i}, i=1, \ldots, k$. Define a weight growth function:

$$
\tilde{\gamma}_{A}(n)=\operatorname{dim}_{K}\left\langle x_{i_{1}} \cdots x_{i_{m}} \mid \operatorname{wt}\left(x_{i_{1}}\right)+\cdots+\operatorname{wt}\left(x_{i_{m}}\right) \leq n, x_{i_{j}} \in X\right\rangle_{K}, \quad n \geq 0 .
$$

Set $C_{1}=\min \left\{\lambda_{i} \mid i=1, \ldots, k\right\}, C_{2}=\max \left\{\lambda_{i} \mid i=1, \ldots, k\right\}$, then $\tilde{\gamma}_{A}\left(C_{1} n\right) \leq \gamma_{A}(n) \leq \tilde{\gamma}_{A}\left(C_{2} n\right)$ for $n \geq 1$. Thus, we obtain an equivalent growth function $\tilde{\gamma}_{A}(n) \sim \gamma_{A}(n)$. Therefore, we can use the weight growth function $\tilde{\gamma}_{A}(n)$ in order to compute the Gelfand-Kirillov dimensions. By $f(n) \approx g(n), n \rightarrow \infty$, denote that $\lim _{n \rightarrow \infty} f(n) / g(n)=1$.

Suppose that $L$ is a Lie (super)algebra and $X \subset L$. By Lie $(X)$ denote the subalgebra of $L$ generated by $X$ (including application of the quadratic mapping in case char $K=2$ ). Let $L$ be a restricted Lie (super)algebra, by $\operatorname{Lie}_{p}(X)$ denote the restricted subalgebra of $L$ generated by $X$. Assume that $X$ is a subset of an associative algebra $A$. Write $\operatorname{Alg}(X) \subset A$ to denote the associative subalgebra (without unit) generated by $X$. A grading of an algebra is called fine if it cannot be splitted by taking a bigger grading group (see definitions in [4, 13]).

Assume that $I$ is a well-ordered set of arbitrary cardinality. Put $\mathbb{Z}_{2}=\{0,1\}$. Let $\mathbb{Z}_{2}^{I}=\left\{\alpha: I \rightarrow \mathbb{Z}_{2}\right\}$ be the set of functions with finitely many nonzero values. Suppose that $\alpha \in \mathbb{Z}_{2}^{I}$ has nonzero values only at $\left\{i_{1}, \ldots, i_{t}\right\} \subset I$, where $i_{1}<\cdots<i_{t}$, put $\mathbf{x}^{\alpha}=x_{i_{1}} x_{i_{2}} \cdots x_{i_{t}}$ and $|\alpha|=t$. Now $\left\{\mathbf{x}^{\alpha} \mid \alpha \in \mathbb{Z}_{2}^{I}\right\}$ is a basis of the Grassmann algebra $\Lambda_{I}=\Lambda\left(x_{i} \mid i \in I\right)$, which is an associative superalgebra $\Lambda_{I}=\Lambda_{\overline{0}} \oplus \Lambda_{\overline{1}}$, all $x_{i}, i \in I$, being odd. Let $\partial_{i}, i \in I$, denote the superderivatives of $\Lambda$ determined by $\partial_{i}\left(x_{j}\right)=\delta_{i j}, i, j \in I$. We identify $x_{i}, i \in I$, with operators of left multiplication on $\Lambda_{I}$, thus we consider that $x_{i} \in \operatorname{End}\left(\Lambda_{I}\right), i \in I$. Consider the space of all formal sums

$$
\mathbf{W}\left(\Lambda_{I}\right)=\left\{\sum_{\alpha \in \mathbb{Z}_{2}^{I}} \mathbf{x}^{\alpha} \sum_{j=1}^{m(\alpha)} \lambda_{\alpha, i_{j}} \partial_{i_{j}} \mid \lambda_{\alpha, i_{j}} \in K, i_{j} \in I\right\} .
$$

It is essential that the sum at each $\mathbf{x}^{\alpha}, \alpha \in \mathbb{Z}_{2}^{I}$, is finite. This construction is similar to so called Lie algebra of special derivations, see [49], [50], [43]. It is similarly verified that the product on $\mathbf{W}\left(\Lambda_{I}\right)$ is well defined and the Lie superalgebra $\mathbf{W}\left(\Lambda_{I}\right)$ acts on $\Lambda_{I}$ by superderivations.

\section{Main Results: Lie superalgebra R And its properties}

Let $\Lambda=\Lambda\left(x_{i} \mid i \geq 0\right)$ be the Grassmann algebra. The Grassmann letters and respective superderivatives $\left\{x_{i}, \partial_{i} \mid i \geq 0\right\}$ are odd elements of the superalgebra End $\Lambda$. One has relations for all $i, j \geq 0$ :

$$
\begin{aligned}
x_{i} x_{j} & =-x_{j} x_{i}, \quad \partial_{i} \partial_{j}=-\partial_{j} \partial_{i}, \quad \partial_{i} x_{j}=-x_{j} \partial_{i}, \quad i \neq j ; \\
x_{i}^{2} & =\partial_{i}^{2}=0 ; \quad \partial_{i} x_{i}+x_{i} \partial_{i}=1 .
\end{aligned}
$$

Our superalgebras will be constructed as subalgebras in $\mathbf{W}(\Lambda) \subset \operatorname{Der} \Lambda$ or $\operatorname{End}(\Lambda)$. Our goal is to study the following finitely generated (restricted) Lie superalgebra and its associative hull.

Example. Let $\Lambda=\Lambda\left(x_{i} \mid i \geq 0\right)$. Consider the following elements in $\mathbf{W}(\Lambda)$ :

$$
v_{i}=\partial_{i}+x_{i} x_{i+1}\left(\partial_{i+2}+x_{i+2} x_{i+3}\left(\partial_{i+4}+x_{i+4} x_{i+5}\left(\partial_{i+6}+\ldots\right)\right)\right), \quad i \geq 0 .
$$

We define a Lie superalgebra $\mathbf{R}=\operatorname{Lie}\left(v_{0}, v_{1}\right) \subset \mathbf{W}(\Lambda)$ and its associative hull $\mathbf{A}=\operatorname{Alg}\left(v_{0}, v_{1}\right) \subset \operatorname{End} \Lambda$. In case char $K=2$, we assume that the quadratic mapping on odd elements is the square of the respective operator in End $\Lambda$.

We call $\left\{v_{i} \mid i \geq 0\right\}$ pivot elements, observe that they are odd in the Lie superalgebra $\mathbf{W}(\Lambda)$. We establish the following properties of $\mathbf{R}=\operatorname{Lie}\left(v_{0}, v_{1}\right)$ and its associative hull $\mathbf{A}=\operatorname{Alg}\left(v_{0}, v_{1}\right)$. 
i) Section 4 yields basic relations of $\mathbf{R}$.

ii) $\mathbf{R}$ has a monomial basis consisting of standard monomials of two types (char $K \neq 2$, Theorem 5.1 ). In case char $K=2$, a basis of $\mathbf{R}$ is given by monomials of the first type and squares of the pivot elements (Corollary 5.3), and $\mathbf{R}$ coincides with the restricted Lie algebra $\operatorname{Lie}_{p}\left(v_{0}, v_{1}\right)$.

iii) We introduce two weight functions wt( ), swt( ) that are additive on products of monomials. Using these functions, we prove that $\mathbf{R}$ and $\mathbf{A}$ are $\mathbb{Z}^{2}$-graded by multidegree in the generators (Lemma 6.3). This allows us to introduce two coordinate systems on plane: multidegree coordinates $\left(X_{1}, X_{2}\right)$ and weight coordinates $\left(Z_{1}, Z_{2}\right)$. We introduce a weight $\mathbb{N}$-gradation and a degree (natural) $\mathbb{N}$-gradation, which components are also the factors of the lower central series (Section 6).

iv) We find bounds on weights and superweights of the basis monomials of $\mathbf{R}$ and $\mathbf{A}$ (Section $\mathbf{7}$ and Section (9) and prove that their monomials are in regions of plane bounded by pairs of logarithmic curves (Theorem 7.4 Figure 1, and Theorem 9.3).

v) The components of the $\mathbb{Z}^{2}$-grading of $\mathbf{R}$ are at most one-dimensional (Theorem $\mathbf{8 . 4}$ ), thus, the $\mathbb{Z}^{2}$ grading of $\mathbf{R}$ is fine. Almost all components of the weight $\mathbb{N}$-gradation of $\mathbf{R}$ are two-dimensional (Corollary 8.3).

vi) $\operatorname{GKdim} \mathbf{R}=\underline{G K \operatorname{dim}} \mathbf{R}=1$, moreover, $\mathbf{R}$ has a linear growth. In case char $K \neq 2$, we establish an asymptotic for the ordinary growth function: $\gamma_{\mathbf{R}}(m) \approx 3 m$, as $m \rightarrow \infty$ (Theorem 8.5).

vii) Moreover, let $\mathbf{R}=\underset{n=1}{\infty} \mathbf{R}_{n}$ be the $\mathbb{N}$-grading by degree in the generators, where also $\mathbf{R}_{n} \cong \mathbf{R}^{n} / \mathbf{R}^{n+1}$, $n \geq 1$, are the lower central series factors. In case char $K \neq 2$, we prove that $\mathbf{R}$ is of finite width 4 , namely, the coefficients $\left(\operatorname{dim} \mathbf{R}_{n} \mid n \geq 1\right.$ ), are $\{2,3,4\}$ (Theorem 11.1). (Recall that both self-similar Lie superalgebras in 41 were of infinite width). This is analogous to the fact that the Grigorchuk group is of finite width [51, 7].

viii) Let char $K=2, \mathbf{R}$ the Lie algebra generated by $\left\{v_{0}, v_{1}\right\}$ (i.e. without $p$-mapping), and $\mathbf{R}=$ $\underset{\oplus=1}{\infty} \mathbf{R}_{n}$ the $\mathbb{N}$-grading by degree in the generators. Then $\mathbf{R}$ is of width 2 . Moreover, the sequence $\left(\operatorname{dim} \mathbf{R}_{n} \mid n \geq 1\right)$, starting with $n \geq 5$, consists of alternating parts of two types: a block 1, 1 followed either by 2 (a diamond) or by 2,2,2 (a triplet of diamonds) (Corollary 11.2). But, $\mathbf{R}$ is not a thin Lie algebra and the sequence above is eventually non-periodic.

ix) Unlike many examples of (self-similar) (restricted) Lie (super)algebras studied before, this is the first example that we are able to find a clear monomial basis of the associative hull $\mathbf{A}$ (Theorem 9.1).

$\mathrm{x}) \operatorname{GKdim} \mathbf{A}=\underline{G K \operatorname{dim}} \mathbf{A}=2$, moreover, we establish quadratic bounds on the growth functions (Theorem 9.4).

xi) We study generating functions of $\mathbf{R}$ (Section 10). The results and proofs on basis monomials of $\mathbf{R}$ are illustrated by Figure 1 and Figure 2 .

xii) As an analogue of the periodicity, we establish that homogeneous elements of the grading $\mathbf{R}=$ $\mathbf{R}_{\overline{0}} \oplus \mathbf{R}_{\overline{1}}$ are ad-nilpotent (Theorem 12.1). We have a triangular decomposition into a direct sum of three locally nilpotent subalgebras $\mathbf{R}=\mathbf{R}_{+} \oplus \mathbf{R}_{0} \oplus \mathbf{R}_{-}$(Theorem 12.3). In case char $K=0$ or char $K=2$, the associative hull $\mathbf{A}$ is not nil; in case char $K=2$, the restricted Lie algebra $\mathbf{R}=\operatorname{Lie}_{p}\left(v_{0}, v_{1}\right)$ is not nil (Lemma 12.4).

xiii) $\mathbf{R}$ is just infinite (Theorem 13.1) but not hereditary just infinite (Lemma 13.2). (Whether two Lie superalgebras of [4] are just infinite is not known).

xiv) $\mathbf{R}$ shows that an extension of Theorem 1.1 (Martinez and Zelmanov [32]) to the Lie superalgebras of characteristic zero is not valid. Such a counterexample of a nil finely $\mathbb{Z}^{3}$-graded Lie superalgebra of slow polynomial growth $\mathbf{Q}$ was suggested before [41. The present example is more handy, because the Lie superalgebra $\mathbf{R}$ is of linear growth, moreover, of finite width 4, and just infinite.

The research is continued in [47, where we study a different just infinite, ad-nil, finely $\mathbb{Z}^{3}$-graded, fractal, 3 -generated Lie superalgebra and suggest new constructions yielding interesting related nil graded fractal Poisson and Jordan superalgebras, that are natural analogues of the Grigorchuk and Gupta-Sidki groups in that classes of algebras.

One can also apply that constructions to the present Lie superalgebra $\mathbf{R}$ and obtain a Jordan superalgebra $\mathbf{K}$, that will be 3 -generated, just infinite, nil $\mathbb{Z}^{3}$-graded, with at most one-dimensional $\mathbb{Z}^{3}$-components, of linear growth, moreover, of finite width 4 , namely, its $\mathbb{N}$-gradation by degree in the generators has a nonperiodic pattern of components of dimensions $\{0,2,3,4\}$, the field being arbitrary with char $K \neq 2$. 


\section{Relations of Lie superalgebra $\mathbf{R}$}

Recall that we consider the Grassmann algebra in infinitely many variables $\Lambda=\Lambda\left(x_{i} \mid i \geq 0\right)$ and its odd derivatives (6) , called the pivot elements. We also present them recursively:

$$
v_{i}=\partial_{i}+x_{i} x_{i+1} v_{i+2}, \quad i \geq 0 .
$$

Below we use a notation $x_{i} \cdots x_{j}=x_{i} x_{i+1} \cdots x_{j-1} x_{j}$ in case $i \leq j$, otherwise the product equals 1 . Let $0 \leq n<m$ and $m-n$ is even. We extend the presentation above as:

$$
v_{n}=\partial_{n}+x_{n} x_{n+1}\left(\partial_{n+2}+x_{n+2} x_{n+3}\left(\partial_{n+4}+\ldots+x_{m-4} x_{m-3}\left(\partial_{m-2}+x_{m-2} x_{m-1} v_{m}\right) \cdots\right)\right) .
$$

Observe that the action of the pivot elements on the Grassmann letters can only produce smaller letters:

$$
v_{n}\left(x_{k}\right)= \begin{cases}0, & k<n ; \\ 1, & k=n ; \\ 0, & k=n+2 l-1, \quad l \geq 1 ; \\ x_{n} x_{n+1} \cdots x_{k-2} x_{k-1}, & k=n+2 l, \quad l \geq 1 .\end{cases}
$$

Define a shift mapping $\tau: \Lambda \rightarrow \Lambda, \tau: \mathbf{W}(\Lambda) \rightarrow \mathbf{W}(\Lambda)$ by $\tau\left(x_{i}\right)=x_{i+1}$ and $\tau\left(\partial_{i}\right)=\partial_{i+1}$ for $i \geq 0$. Clearly, $\tau$ is an endomorphism such that $\tau\left(v_{i}\right)=v_{i+1}, i \geq 0$.

First, we obtain relations between neighbor pivot elements.

Lemma 4.1. We have the following relations, $i \geq 0$ :

i) $v_{i}^{2}=x_{i+1} v_{i+2}$;

ii) $\left[v_{i}, v_{i}\right]=2 x_{i+1} v_{i+2}$;

iii) $\left[v_{i}, v_{i+1}\right]=-x_{i} v_{i+2}$;

iv) $\left[v_{i},\left[v_{i}, v_{i+1}\right]\right]=\left[v_{i}^{2}, v_{i+1}\right]=-v_{i+2}$;

v) $\left[v_{i}, v_{i+2}\right]=2 x_{i} x_{i+1} x_{i+3} v_{i+4}$.

Proof. To prove (i) we use (7) and (4):

$$
v_{i}^{2}=\left(\partial_{i}+x_{i} x_{i+1} v_{i+2}\right)^{2}=\partial_{i}^{2}+\left(x_{i} x_{i+1} v_{i+2}\right)^{2}+\left[\partial_{i}, x_{i} x_{i+1} v_{i+2}\right]=x_{i+1} v_{i+2} .
$$

Now (ii) follows by $\left[v_{i}, v_{i}\right]=2 v_{i}^{2}$. The remaining claims are checked below

$$
\begin{aligned}
{\left[v_{i}, v_{i+1}\right] } & =\left[\partial_{i}+x_{i} x_{i+1} v_{i+2}, \partial_{i+1}+x_{i+1} x_{i+2} v_{i+3}\right]=\left[x_{i} x_{i+1} v_{i+2}, \partial_{i+1}\right]=-x_{i} v_{i+2} ; \\
{\left[v_{i}^{2}, v_{i+1}\right] } & =\left[v_{i},\left[v_{i}, v_{i+1}\right]\right]=\left[\partial_{i}+x_{i} x_{i+1} v_{i+2},-x_{i} v_{i+2}\right]=-v_{i+2} ; \\
{\left[v_{i}, v_{i+2}\right] } & =\left[\partial_{i}+x_{i} x_{i+1} v_{i+2}, v_{i+2}\right]=x_{i} x_{i+1}\left[v_{i+2}, v_{i+2}\right]=2 x_{i} x_{i+1} x_{i+3} v_{i+4} .
\end{aligned}
$$

Consider general products of the pivot elements.

Lemma 4.2. For any integers $i, k \geq 0$, we have:

i) if $k$ is even, then $\left[v_{i}, v_{i+k}\right]=2 x_{i} \cdots x_{i+k-1} x_{i+k+1} v_{i+k+2}$;

ii) if $k$ is odd, then $\left[v_{i}, v_{i+k}\right]=-x_{i} \cdots x_{i+k-1} v_{i+k+1}$.

Proof. Let $k$ be even, we use item (ii) of Lemma 4.1, and expanded presentation (8):

$$
\begin{aligned}
{\left[v_{i}, v_{i+k}\right] } & =\left[\partial_{i}+x_{i} x_{i+1} \partial_{i+2}+\ldots+x_{i} \cdots x_{i+k-3} \partial_{i+k-2}+x_{i} \cdots x_{i+k-1} v_{i+k}, v_{i+k}\right] \\
& =x_{i} \cdots x_{i+k-1}\left[v_{i+k}, v_{i+k}\right]=2 x_{i} \cdots x_{i+k-1} x_{i+k+1} v_{i+k+2} .
\end{aligned}
$$

Let $k$ be odd. We use item (iii) of Lemma 4.1

$$
\begin{aligned}
{\left[v_{i}, v_{i+k}\right] } & =\left[\partial_{i}+x_{i} x_{i+1} \partial_{i+2}+\ldots+x_{i} \cdots x_{i+k-4} \partial_{i+k-3}+x_{i} \cdots x_{i+k-2} v_{i+k-1}, v_{i+k}\right] \\
& =x_{i} \cdots x_{i+k-2}\left[v_{i+k-1}, v_{i+k}\right]=-x_{i} \cdots x_{i+k-1} v_{i+k+1} .
\end{aligned}
$$

Consider Lie superalgebras generated by two consecutive pivot elements: $L_{i}=\operatorname{Lie}\left(v_{i}, v_{i+1}\right), i \geq 0$, in particular, $L_{0}=\mathbf{R}$.

Lemma 4.3. Consider the Lie superalgebra $\mathbf{R}=\operatorname{Lie}\left(v_{0}, v_{1}\right)$. We have

i) $v_{i} \in \mathbf{R}, i \geq 0$ (moreover, we get these elements using Lie bracket only in case of arbitrary $K$ );

ii) $\tau^{i}: \mathbf{R} \rightarrow L_{i}$ is an isomorphism for all $i \geq 0$;

iii) $\mathbf{R}$ is infinite dimensional.

Proof. We use claim (iv) of Lemma 4.1. 
The notion of self-similarity plays an important role in group theory [22, 36. The Fibonacci Lie algebra is "self-similar" 44 but not in terms of the definition of self-similarity given by Bartholdi [6]. Namely, a Lie algebra $L$ is called self-similar if it affords a homomorphism [6]:

$$
\psi: L \rightarrow \operatorname{Der} R \curlywedge(R \otimes L),
$$

where $R$ is a commutative algebra, Der $R$ its Lie algebra of derivations naturally acting on $R$. This definition easily extends to Lie superalgebras, namely, one considers $R$ to be a supercommutative associative superalgebra. For example, a self-similarity embedding should look like:

$$
\mathbf{R} \hookrightarrow\left\langle\partial_{0}\right\rangle_{K} \curlywedge\left(\Lambda\left(x_{0}\right) \otimes \tau(\mathbf{R})\right) .
$$

Unlike two examples of self-similar Lie superalgebras [41] and a family that includes many nil self-similar restricted Lie algebras [42, it seems that such a self-similarity embedding for $\mathbf{R}$ does not exist.

\section{Monomial basis of Lie superalgebra $\mathbf{R}$}

Now our goal is to describe a clear monomial basis for the (restricted) Lie superalgebra $\mathbf{R}=\operatorname{Lie}\left(v_{0}, v_{1}\right)$ for arbitrary field $K$. Let us introduce a notation widely used below. By $r_{n}$ denote a tail monomial:

$$
r_{n}=x_{0}^{\xi_{0}} \cdots x_{n}^{\xi_{n}} \in \Lambda, \quad \xi_{i} \in\{0,1\} ; \quad n \geq 0 .
$$

For $n<0$ we assume that $r_{n}=1$. If needed, other monomials of type (10) will be denoted by $r_{n}^{\prime}, r_{n}^{\prime \prime}$, etc.

Theorem 5.1. Let char $K \neq 2$. A basis of the Lie superalgebra $\mathbf{R}=\operatorname{Lie}\left(v_{0}, v_{1}\right)$ is given by

i) standard monomials of the first type:

$$
\left\{r_{n-2} v_{n} \mid n \geq 0\right\}
$$

ii) standard monomials of the second type:

$$
\left\{r_{n-3} x_{n-1} v_{n} \mid n \geq 2\right\} \backslash\left\{x_{0} x_{2} v_{3}\right\} .
$$

Proof. Let us call $n$ a length, $v_{n}$ a head, $r_{n-2}\left(r_{n-3}\right.$, sometimes, $\left.r_{n-3} x_{n-1}\right)$ a tail, and the optional letter $x_{n-1}$ a neck of the monomial. We call $x_{0} x_{2} v_{3}$ a false monomial.

First, we prove that our monomials belong to $\mathbf{R}$. We show by induction on length $n$ that all monomials of the first type belong to $\mathbf{R}$. Clearly, such monomials of lengths $n=0,1$, namely, $v_{0}, v_{1}$, belong to $\mathbf{R}$. Consider $n \geq 2$, by inductive assumption, all elements $r_{k-2} v_{k}$ with $0 \leq k \leq n-1$ belong to $\mathbf{R}$. Consider

$$
\begin{aligned}
& {\left[v_{n-2}^{2}, r_{n-3} v_{n-1}\right]=r_{n-3}\left[v_{n-2}^{2}, v_{n-1}\right]=-r_{n-3} v_{n},} \\
& {\left[v_{n-2}, r_{n-3} v_{n-1}\right]= \pm r_{n-3}\left[v_{n-2}, v_{n-1}\right]=\mp r_{n-3} x_{n-2} v_{n} .}
\end{aligned}
$$

We conclude that all monomials of the first type of length $n$ belong to $\mathbf{R}$.

Consider monomials of the second type. By $\left[v_{0}, v_{0}\right]=2 x_{1} v_{2},\left[v_{1}, v_{1}\right]=2 x_{2} v_{3}$, we obtain all such monomials of lengths 2,3 . Let $n \geq 4$. Depending on parity of $n$, using Lemma 4.2 we get

$$
\begin{array}{rlrl}
{\left[v_{0}, v_{n-2}\right]} & =2 x_{0} \cdots x_{n-3} x_{n-1} v_{n} \in \mathbf{R}, & n \text { even } \\
{\left[v_{1}, x_{0} v_{n-2}\right]} & =-x_{0}\left[v_{1}, v_{n-2}\right]=-2 x_{0} x_{1} \cdots x_{n-3} x_{n-1} v_{n} \in \mathbf{R}, \quad n \text { odd } .
\end{array}
$$

Multiplying these elements by $v_{i}$, where $0 \leq i \leq n-3$, we can delete any factors $x_{i}$ above. We conclude that all monomials of the second type of length $n$ belong to $\mathbf{R}$.

Second, we prove that commutators of the standard monomials are expressed via the standard monomials. Some efforts below are necessary to verify that the false monomial $x_{0} x_{2} v_{3}$ shall not appear. Sometimes, we write standard monomials as $r_{n-1} v_{n}$.

A) Consider products of standard monomials of the same length $n$.

$$
\left[r_{n-1} v_{n}, r_{n-1}^{\prime} v_{n}\right]= \pm 2 r_{n-1}^{\prime \prime} x_{n+1} v_{n+2},
$$

a standard monomial of the second type, except eventually for $n=1$. But the only possible such product is $\left[v_{1}, v_{1}\right]=2 x_{2} v_{3}$, and the false monomial is not possible. The false monomial cannot appear here and below by weight arguments, see below Lemma 6.7

B) Consider two standard monomials of different lengths $u=r_{n-1} v_{n}, v=r_{m-1}^{\prime} v_{m}$, where $n<m$. 
B1) Assume that $n \equiv m(\bmod 2)$. We use presentation (8):

$$
\begin{aligned}
{[u, v]=} & {\left[r_{n-1} v_{n}, r_{m-1}^{\prime} v_{m}\right] } \\
= & {\left[r_{n-1}\left(\partial_{n}+x_{n} x_{n+1} \partial_{n+2}+\ldots+x_{n} \cdots x_{m-3} \partial_{m-2}+x_{n} \cdots x_{m-1} v_{m}\right), r_{m-1}^{\prime} v_{m}\right] } \\
= & r_{n-1}\left(\partial_{n}\left(r_{m-1}^{\prime}\right)+x_{n} x_{n+1} \partial_{n+2}\left(r_{m-1}^{\prime}\right)+\ldots+x_{n} \cdots x_{m-3} \partial_{m-2}\left(r_{m-1}^{\prime}\right)\right) v_{m} \\
& \pm r_{n-1} x_{n} \cdots x_{m-1} r_{m-1}^{\prime}\left[v_{m}, v_{m}\right] .
\end{aligned}
$$

The last summand equals $\pm 2 r_{m-1}^{\prime \prime} x_{m+1} v_{m+2}$, which is of the second type. Assume that $v$ is of the first type, namely, $v=r_{m-1}^{\prime} v_{m}=r_{m-2}^{\prime \prime} v_{m}$, then all monomials in (11) are of the first type. Assume that $v$ is of the second type, namely, $v=r_{m-1}^{\prime} v_{m}=r_{m-3}^{\prime \prime} x_{m-1} v_{m}$, then all summands (11) are monomials of the second type because the derivatives cannot remove the letter $x_{m-1}$ while the remaining factors yield $\tilde{r}_{m-3}$.

B2) Assume that $n \not \equiv m(\bmod 2)$. Again, we use (8) :

$$
\begin{aligned}
{[u, v]=} & {\left[r_{n-1} v_{n}, r_{m-1}^{\prime} v_{m}\right] } \\
= & {\left[r_{n-1}\left(\partial_{n}+x_{n} x_{n+1} \partial_{n+2}+\ldots+x_{n} \cdots x_{m-4} \partial_{m-3}+x_{n} \cdots x_{m-2} v_{m-1}\right), r_{m-1}^{\prime} v_{m}\right] } \\
= & r_{n-1}\left(\partial_{n}\left(r_{m-1}^{\prime}\right)+x_{n} x_{n+1} \partial_{n+2}\left(r_{m-1}^{\prime}\right)+\ldots+x_{n} \cdots x_{m-4} \partial_{m-3}\left(r_{m-1}^{\prime}\right)\right) v_{m} \\
& \pm r_{n-1} x_{n} \cdots x_{m-2} r_{m-1}^{\prime}\left[v_{m-1}, v_{m}\right] .
\end{aligned}
$$

The last summand equals $\mp \tilde{r}_{m-1} x_{m-1} v_{m+1}$, which is of the first type. The remaining monomials have the same type as that of $v$ by the arguments above.

Corollary 5.2. Let char $K=0$. Consider $\operatorname{Lie}_{\mathbb{Z}}\left(v_{0}, v_{1}\right)$, the Lie super-subring in $\mathbf{R}=\operatorname{Lie}\left(v_{0}, v_{1}\right)$, generated by $v_{0}, v_{1}$ using coefficients in $\mathbb{Z}$. It has the following $\mathbb{Z}$-basis:

i) standard monomials of the first type;

ii) $\left\{x_{n-1} v_{n} \mid n \geq 2\right\}$ (squares of the pivot elements);

iii) $\left\{2 r_{n-3} x_{n-1} v_{n} \mid r_{n-3} \neq 1, r_{n-3} x_{n-1} v_{n} \neq x_{0} x_{2} v_{3}, n \geq 2\right\}$ (2-multiples of the remaining standard monomials of the second type).

Proof. The result follows from computations above. The axioms of a Lie-super ring include the formal square (Section 2) and yield the second claim.

In case $p=2$ superderivatives are ordinary derivatives and our example yields also a Lie algebra.

Corollary 5.3. Let char $K=p=2$.

i) $A$ basis of the Lie algebra $\mathbf{R}=\operatorname{Lie}\left(v_{0}, v_{1}\right)$ is given by monomials of the first type.

ii) $A$ basis of the Lie superalgebra $\mathbf{R}=\operatorname{Lie}\left(v_{0}, v_{1}\right)$, as well as a basis of the restricted Lie (super)algebra $\operatorname{Lie}_{p}\left(v_{0}, v_{1}\right)$ is given by

(a) standard monomials of the first type;

(b) $\left\{x_{n-1} v_{n} \mid n \geq 2\right\}$ (squares of the pivot elements).

Proof. Let $L=L_{\overline{0}} \oplus L_{\overline{1}} \subset \mathbf{W}(\Lambda)$ be the Lie subalgebra generated by $v_{0}, v_{1}$ (i.e. we use the bracket [ , ] only). By proof of Theorem [5.1, its basis $\left\{w_{j} \mid j \in J\right\}$ consists of monomials of the first type, thus yielding the first claim.

In order to obtain the restricted Lie algebra $\operatorname{Lie}_{p}\left(v_{0}, v_{1}\right)$, which coincides with the $p$-hull $\operatorname{Lie}_{p}(L)$, it is sufficient to add all $p^{n}$-powers of basis elements: $\operatorname{Lie}_{p}(L)=\left\langle w_{j}^{\left[p^{n}\right]} \mid n \geq 1, j \in J\right\rangle_{K}$ [60]. These powers are trivial except squares of the pivot elements. The same observation holds in case of the restricted Lie superalgebra.

Consider the case of the Lie superalgebra $\mathbf{R}=\operatorname{Lie}\left(v_{0}, v_{1}\right)$. Now, we need to add squares of a basis of the odd component $L_{\overline{1}}$. Again, we add the same squares of the pivot elements.

Corollary 5.4. Let char $K=p>2$. A basis of the restricted Lie superalgebra $\operatorname{Lie}_{p}\left(v_{0}, v_{1}\right)$ is the same as that for the Lie superalgebra $\mathbf{R}$ described in Theorem [5.1.

Proof. Powers of the even basis elements of $\operatorname{Lie}\left(v_{0}, v_{1}\right)$ are trivial because they contain Grassmann letters. 


\section{Weight Functions, $\mathbb{Z}^{2}$-GRAdation, And two COORDinAte SYstems}

In this section we introduce two weight functions. Using them we establish that our algebras are $\mathbb{Z}^{2}$-graded my multidegree in the generators and derive further corollaries.

We start with the Lie superalgebra $\mathbf{W}\left(\Lambda_{I}\right)$ of special superderivations of the Grassmann algebra $\Lambda_{I}=$ $\Lambda\left(x_{i} \mid i \in I\right)$ and consider a subalgebra spanned by pure Lie monomials:

$$
\mathbf{W}_{\text {fin }}\left(\Lambda_{I}\right)=\left\langle x_{i_{1}} \cdots x_{i_{m}} \partial_{j} \mid i_{k}, j \in I\right\rangle_{K} \subset \mathbf{W}\left(\Lambda_{I}\right) .
$$

Define a weight function on the Grassmann variables and respective superderivatives related as:

$$
w t\left(\partial_{i}\right)=-w t\left(x_{i}\right)=\alpha_{i} \in \mathbb{C}, \quad i \geq 0,
$$

and extend it to pure Lie monomials $w t\left(x_{i_{1}} \cdots x_{i_{m}} \partial_{j}\right)=-\alpha_{i_{1}}-\cdots-\alpha_{i_{m}}+\alpha_{j}, i_{k}, j \in I$. One checks that the weight function is additive, namely, $w t\left(\left[w_{1}, w_{2}\right]\right)=w t\left(w_{1}\right)+w t\left(w_{2}\right)$, where $w_{1}, w_{2}$ are pure Lie monomials. The weight function is also extended to an associative hull $\operatorname{Alg}\left(\mathbf{W}_{\text {fin }}\left(\Lambda_{I}\right)\right)$ and it is additive on associative products of its monomials.

Now we return to $\mathbf{R}=\operatorname{Lie}\left(v_{0}, v_{1}\right)$ and $\mathbf{A}=\operatorname{Alg}\left(v_{0}, v_{1}\right)$. We want all terms in (7) to have the same weight. Namely, assume that $w t\left(v_{i}\right)=w t\left(\partial_{i}\right)=\alpha_{i}=-\alpha_{i}-\alpha_{i+1}+\alpha_{i+2}, i \geq 0$. We get a recurrence relation

$$
\alpha_{i+2}=\alpha_{i+1}+2 \alpha_{i}, \quad i \geq 0 .
$$

The roots of the respective characteristic polynomial are $\lambda_{1}=2$ and $\lambda_{2}=-1$.

Lemma 6.1. A base of the space of solutions of the recurrence equation (12) is given by two weight functions $\mathrm{wt}($ ) and $\mathrm{swt}()$ defined as follows:

i) $\operatorname{wt}\left(\partial_{n}\right)=\operatorname{wt}\left(v_{n}\right)=-\operatorname{wt}\left(x_{n}\right)=2^{n}, n \geq 0$ (the weight function);

ii) $\operatorname{swt}\left(\partial_{n}\right)=\operatorname{swt}\left(v_{n}\right)=-\operatorname{swt}\left(x_{n}\right)=(-1)^{n}, n \geq 0$ (the superweight function);

iii) we combine these functions together as $\mathrm{Wt}\left(v_{n}\right)=\left(\operatorname{wt}\left(v_{n}\right), \operatorname{swt}\left(v_{n}\right)\right)=\left(2^{n},(-1)^{n}\right), n \geq 0$, (the vector weight function).

Below, a monomial is any (Lie or associative) product of letters $\left\{x_{i}, \partial_{i}, v_{i} \mid i \geq 0\right\} \subset$ End $\Lambda$. By arguments above, we have the following.

Lemma 6.2. The weight functions are well defined on monomials. They are additive on (Lie or associative) products of monomials, e.g., $\mathrm{Wt}(a \cdot b)=\mathrm{Wt}(a)+\mathrm{Wt}(b)$, where $a, b$ are monomials of $\mathbf{A}$.

As a first application we establish $\mathbb{Z}^{2}$-gradations.

Lemma 6.3. The algebras $\mathbf{R}=\operatorname{Lie}\left(v_{0}, v_{1}\right)$ and $\mathbf{A}=\operatorname{Alg}\left(v_{0}, v_{1}\right)$ are $\mathbb{Z}^{2}$-graded by multidegree in $\left\{v_{0}, v_{1}\right\}$ :

$$
\mathbf{R}=\underset{n_{1}, n_{2} \geq 0}{\bigoplus} \mathbf{R}_{n_{1}, n_{2}}, \quad \mathbf{A}=\underset{n_{1}, n_{2} \geq 0}{\bigoplus} \mathbf{A}_{n_{1}, n_{2}} .
$$

Proof. Consider the weight vectors for the generators of our algebras $\mathrm{Wt}\left(v_{0}\right)=(1,1)$ and $\mathrm{Wt}\left(v_{1}\right)=(2,-1)$ given by Lemma 6.1. For $n_{1}, n_{2} \geq 0$, let $\mathbf{R}_{n_{1} n_{2}} \subset \mathbf{R}$ be the subspace spanned by all Lie elements of multidegree $\left(n_{1}, n_{2}\right)$ in $\left\{v_{0}, v_{1}\right\}$. By Lemma 6.2, all elements $v \in \mathbf{R}_{n_{1} n_{2}}$ have the same vector weight:

$$
\mathrm{Wt}(v)=n_{1} \mathrm{Wt}\left(v_{0}\right)+n_{2} \mathrm{Wt}\left(v_{1}\right)=\left(n_{1}+2 n_{2}, n_{1}-n_{2}\right) .
$$

Elements of $\mathbf{R}_{n_{1} n_{2}} \subset \mathbf{W}\left(\Lambda_{I}\right)$ are written as (probably infinite) linear combinations of pure Lie monomials. Since the vectors $\mathrm{Wt}\left(v_{0}\right), \mathrm{Wt}\left(v_{1}\right)$ are linearly independent, two different components $\mathbf{R}_{n_{1}, n_{2}}, \mathbf{R}_{n_{1}^{\prime}, n_{2}^{\prime}}$ have different weights, hence they are expressed via different sets of pure Lie monomials. We conclude that the sum of the components is direct. The $\mathbb{Z}^{2}$-gradation follows by definition of these components.

Let $v \in \mathbf{A}_{n_{1} n_{2}}$, then $\operatorname{swt}(v)=n_{1} \operatorname{swt}\left(v_{0}\right)+n_{2} \operatorname{swt}\left(v_{1}\right)=n_{1}-n_{2}$. Now, $\operatorname{swt}(v)=0$ if and only if $n_{1}=n_{2}$. We call $\underset{n \geq 0}{\oplus} \mathbf{A}_{n n}$ a diagonal of the $\mathbb{Z}^{2}$-gradation of $\mathbf{A}$. Similarly, $\underset{n \geq 0}{\oplus} \mathbf{R}_{n n}$ is a diagonal of the $\mathbb{Z}^{2}$-gradation of $\mathbf{R}$. The additivity of the superweight function easily implies the following fact.

Corollary 6.4. The following statements are valid:

i) The algebras $\mathbf{R}$ and $\mathbf{A}$ allow triangular decompositions into direct sums of three subalgebras:

$$
\mathbf{R}=\mathbf{R}_{+} \oplus \mathbf{R}_{0} \oplus \mathbf{R}_{-}, \quad \mathbf{A}=\mathbf{A}_{+} \oplus \mathbf{A}_{0} \oplus \mathbf{A}_{-},
$$

where the respective components are spanned by monomials of positive, zero, and negative superweights.

ii) The zero components above coincide with the respective diagonals:

$$
\mathbf{R}_{0}=\underset{n \geq 0}{\bigoplus} \mathbf{R}_{n n}, \quad \mathbf{A}_{0}=\underset{n \geq 0}{\bigoplus} \mathbf{A}_{n n} .
$$


Given a nonzero homogeneous element $v \in \mathbf{A}_{n_{1} n_{2}}, n_{1}, n_{2} \geq 0$, we define its multidegree vector and degree:

$$
\operatorname{Gr}(v)=\left(n_{1}, n_{2}\right) \in \mathbb{Z}^{2} \subset \mathbb{R}^{2}, \quad \operatorname{deg}(v)=n_{1}+n_{2} .
$$

We put it on plane using standard coordinates $\left(X_{1}, X_{2}\right) \in \mathbb{R}^{2}$, which we also call multidegree coordinates. Thus, we write $\operatorname{Gr}(v)=\left(n_{1}, n_{2}\right)=\left(X_{1}, X_{2}\right)$.

Let $\left(X_{1}, X_{2}\right) \in \mathbb{R}^{2}$ be an arbitrary point of plane in terms of the standard coordinates. We introduce its weight coordinates $\left(Z_{1}, Z_{2}\right)$ :

$$
\left\{\begin{array} { l } 
{ Z _ { 1 } = X _ { 1 } + 2 X _ { 2 } ; } \\
{ Z _ { 2 } = X _ { 1 } - X _ { 2 } ; }
\end{array} \quad \left\{\begin{array}{l}
X_{1}=\left(Z_{1}+2 Z_{2}\right) / 3 \\
X_{2}=\left(Z_{1}-Z_{2}\right) / 3
\end{array}\right.\right.
$$

Lemma 6.5. Let $v \in \mathbf{A}$ be a monomial, $\operatorname{Gr}(v)=\left(n_{1}, n_{2}\right)=\left(X_{1}, X_{2}\right)$ its multidegree, and $\left(Z_{1}, Z_{2}\right)$ the respective weight coordinates. Then

$$
\left(Z_{1}, Z_{2}\right)=\operatorname{Wt}(v)=(\operatorname{wt}(v), \operatorname{swt}(v)) .
$$

Proof. By (13) and (14), we have

$$
\begin{aligned}
\operatorname{wt}(v) & =n_{1}+2 n_{2}=X_{1}+2 X_{2}=Z_{1}, \\
\operatorname{swt}(v) & =n_{1}-n_{2}=X_{1}-X_{2}=Z_{2} .
\end{aligned}
$$

Lemma 6.6. Consider the pivot elements $\left\{v_{n} \mid n \geq 0\right\}$. Then

i) $\operatorname{Gr}\left(v_{n}\right)=\frac{1}{3}\left(2^{n}+2(-1)^{n}, 2^{n}-(-1)^{n}\right), \quad n \geq 0$;

ii) The pivot elements belong to two parallel lines: $X_{1}-X_{2}=1$ and $X_{1}-X_{2}=-1$.

Proof. By Lemma 6.1. Wt $\left(v_{n}\right)=\left(2^{n},(-1)^{n}\right)=\left(Z_{1}, Z_{2}\right)$, we use (14) and obtain the first claim. Observe that $X_{1}-X_{2}=Z_{2}=\operatorname{swt}\left(v_{n}\right)=(-1)^{n}$.

Lemma 6.7. Consider the false monomial $u=x_{0} x_{2} v_{3}$.

i) $\operatorname{Gr}(u)=(-1,2)$;

ii) the false monomial cannot appear as a homogeneous component of an element of $\mathbf{R}$.

Proof. We use Lemma 6.1 and (14)

$$
\begin{aligned}
& \operatorname{Wt}(u)=\left(Z_{1}, Z_{2}\right)=\operatorname{Wt}\left(v_{3}\right)-\mathrm{Wt}\left(v_{2}\right)-\operatorname{Wt}\left(v_{0}\right)=(8,-1)-(4,1)-(1,1)=(3,-3) ; \\
& \operatorname{Gr}(u)=\left(Z_{1}+2 Z_{2}, Z_{1}-Z_{2}\right) / 3=(-1,2) .
\end{aligned}
$$

Any homogeneous element of $\mathbf{R}=\operatorname{Lie}\left(v_{0}, v_{1}\right)$ must have nonnegative multidegree coordinates in the generators. Therefore, $v=x_{0} x_{2} v_{3}$ cannot appear.

Lemma 6.8. Let $0 \neq v \in \mathbf{A}_{n_{1} n_{2}}, n_{1}, n_{2} \geq 0$, and $\tau(v)$ the image under the endomorphism $\tau$. Then

$$
\operatorname{Gr}(\tau(v))=\left(2 n_{2}, n_{1}+n_{2}\right) .
$$

Proof. The relation $\left[v_{0}^{2}, v_{1}\right]=-v_{2}$ implies that $\operatorname{Gr}\left(v_{2}\right)=(2,1)$. By assumption, $v$ is a linear combination of products involving $n_{1}$ factors $v_{0}$ and $n_{2}$ factors $v_{1}$. Since $\tau$ is an endomorphism, $\tau(v)$ is a linear combination of products involving $n_{1}$ factors $\tau\left(v_{0}\right)$ and $n_{2}$ factors $\tau\left(v_{1}\right)$. Using $\tau\left(v_{0}\right)=v_{1}, \tau\left(v_{1}\right)=v_{2}$, and additivity of the multidegree function, we get

$$
\operatorname{Gr}(\tau(v))=n_{1} \operatorname{Gr}\left(v_{1}\right)+n_{2} \operatorname{Gr}\left(v_{2}\right)=n_{1}(0,1)+n_{2}(2,1)=\left(2 n_{2}, n_{1}+n_{2}\right) .
$$

The multidegree $\mathbb{Z}^{2}$-gradation induces a weight $\mathbb{N}$-gradation:

$$
\left.\mathbf{R}=\bigoplus_{n=1}^{\infty} \tilde{\mathbf{R}}_{n}, \quad \tilde{\mathbf{R}}_{n}=\left\langle w \in \mathbf{R}_{n_{1}, n_{2}}\right| \text { wt } w=n_{1}+2 n_{2}=n\right\rangle_{K}, \quad n \geq 1 .
$$

Similarly, one defines a superweight $\mathbb{Z}$-gradation:

$$
\left.\mathbf{R}=\underset{n=-\infty}{+\infty} \mathbf{R}_{n}^{\prime}, \quad \mathbf{R}_{n}^{\prime}=\left\langle w \in \mathbf{R}_{n_{1}, n_{2}}\right| \text { swt } w=n_{1}-n_{2}=n\right\rangle_{K}, \quad n \in \mathbb{Z}
$$

Finally, we have a degree $\mathbb{N}$-gradation:

$$
\mathbf{R}=\bigoplus_{n=1}^{\infty} \mathbf{R}_{n}, \quad \mathbf{R}_{n}=\left\langle w \in \mathbf{R}_{n_{1}, n_{2}} \mid \operatorname{deg} w=n_{1}+n_{2}=n\right\rangle_{K}, \quad n \geq 1 .
$$

The last gradation is important to us because it is related to the lower central series.

Lemma 6.9. The terms of the lower central series of $\mathbf{R}$ are as follows:

i) $\mathbf{R}^{m}=\underset{n \geq m}{\oplus} \mathbf{R}_{n}, m \geq 1$; 
ii) the lower central series factors are isomorphic to the terms of the degree $\mathbb{N}$-gradation: $\mathbf{R}^{n} / \mathbf{R}^{n+1} \cong$ $\mathbf{R}_{n}$, for all $n \geq 1$;

iii) let char $K \neq 2$, then $\operatorname{dim} \mathbf{R} / \mathbf{R}^{m} \approx 3 m$, as $m \rightarrow \infty$;

iv) let char $K=2$, then $\operatorname{dim} \mathbf{R} / \mathbf{R}^{m} \approx 3 m / 2$, as $m \rightarrow \infty$.

Proof. Recall that the multidegree component $\mathbf{R}_{n_{1} n_{2}}$ is spanned by commutators containing $n_{1}$ factors $v_{0}$ and $n_{2}$ factors $v_{1}$. On the other hand, the term of the lower central series $\mathbf{R}^{m}, m \geq 1$, is spanned by all commutators of length at least $m$ in the generators $\left\{v_{0}, v_{1}\right\}[1]$, thus $\mathbf{R}^{m}=\underset{n_{1}+n_{2} \geq m}{\oplus} \mathbf{R}_{n_{1} n_{2}}=\underset{n \geq m}{\oplus} \mathbf{R}_{n}$.

By Theorem 8.5, using the ordinary growth function we have $\operatorname{dim} \mathbf{R} / \mathbf{R}^{m}=\operatorname{dim}\left(\mathbf{R}_{1} \oplus \cdots \oplus \mathbf{R}_{m-1}\right)=$ $\gamma_{\mathbf{R}}(m-1) \approx 3 m, m \rightarrow \infty$ in the case char $K \neq 2$.

Corollary 6.10. $\mathbf{R}$ is a naturally graded algebra with respect to the degree $\mathbb{N}$-gradation in the sense [35], namely, the associated graded algebra related to the filtration by the lower central series $\left(\mathbf{R}^{m} \mid m \geq 1\right)$ is isomorphic to the original $\mathbb{N}$-graded algebra $\mathbf{R}=\underset{n=1}{\infty} \mathbf{R}_{n}$.

\section{Bounds on Weights of MONOMials of Lie SUPERALGEBRA $\mathbf{R}$}

In this section we establish estimates on weights and superweights of the standard monomials of the Lie superalgebra $\mathbf{R}$, which allow us to prove that the standard monomials are situated in a region of plane restricted by two logarithmic curves (Theorem 7.4).

Lemma 7.1. Weights of standard monomials of the first and second type satisfy the following inequalities:

$$
\begin{array}{ll}
2^{n-1}<\operatorname{wt}\left(r_{n-2} v_{n}\right) \leq 2^{n}, & n \geq 0 ; \\
2^{n-2}<\operatorname{wt}\left(r_{n-3} x_{n-1} v_{n}\right) \leq 2^{n-1}, & n \geq 2 .
\end{array}
$$

Proof. First, observe that weight of a tail $r_{m}=x_{0}^{\xi_{0}} \cdots x_{m}^{\xi_{m}}, \xi_{i} \in\{0,1\}$, has the following bounds:

$$
0 \geq \operatorname{wt}\left(r_{m}\right) \geq-\left(2^{0}+2^{1}+\cdots+2^{m}\right)=-2^{m+1}+1, \quad m \geq 0 .
$$

Theses bounds are also formally valid for $m=-1$. Using these bounds we obtain:

$$
2^{n} \geq \operatorname{wt}\left(r_{n-2} v_{n}\right) \geq-2^{n-1}+1+2^{n}=2^{n-1}+1>2^{n-1}, \quad n \geq 1 .
$$

The outside bounds above are also valid for $n=0$. Let us check bounds for monomials of the second type:

$$
2^{n-1}=2^{n}-2^{n-1} \geq \operatorname{wt}\left(r_{n-3} x_{n-1} v_{n}\right) \geq-2^{n-2}+1-2^{n-1}+2^{n}=2^{n-2}+1>2^{n-2}, \quad n \geq 2 .
$$

Lemma 7.2. Superweights of standard monomials have the following bounds:

$$
\begin{array}{ll}
-\frac{n}{2}-\frac{1}{2} \leq \operatorname{swt}\left(r_{n-2} v_{n}\right) \leq \frac{n}{2}, & n \geq 1 ; \\
-\frac{n}{2}-\frac{3}{2} \leq \operatorname{swt}\left(r_{n-3} x_{n-1} v_{n}\right) \leq \frac{n}{2}+1, & n \geq 2 .
\end{array}
$$

Proof. Let $w=r_{n-2} v_{n}, n \geq 1$. Consider the case $n=2 k, k \geq 1$. We have $r_{n-2}=x_{0}^{\xi_{0}} x_{1}^{\xi_{1}} \cdots x_{2 k-3}^{\xi_{2 k-3}} x_{2 k-2}^{\xi_{2 k-2}}$, $0 \leq \xi_{i} \leq 1$, where $\operatorname{swt}\left(x_{0}\right)=\operatorname{swt}\left(x_{2}\right)=\cdots=\operatorname{swt}\left(x_{2 k-2}\right)=-1, \operatorname{swt}\left(x_{1}\right)=\operatorname{swt}\left(x_{3}\right)=\cdots=\operatorname{swt}\left(x_{2 k-3}\right)=1$, and $\operatorname{swt}\left(v_{n}\right)=1$. We get:

$$
\begin{aligned}
-k & \leq \operatorname{swt}\left(r_{n-2}\right) \leq k-1 ; \\
-\frac{n}{2}+1=-k+1 & \leq \operatorname{swt}\left(r_{n-2} v_{n}\right) \leq k=\frac{n}{2} .
\end{aligned}
$$

Consider the case $n=2 k+1, k \geq 0$. Similarly, we get

$$
\begin{aligned}
-k & \leq \operatorname{swt}\left(r_{n-2}\right) \leq k ; \\
-\frac{n}{2}-\frac{1}{2}=-k-1 & \leq \operatorname{swt}\left(r_{n-2} v_{n}\right) \leq k-1=\frac{n}{2}-\frac{3}{2} .
\end{aligned}
$$

These cases yield the claimed bounds for monomials of the first type.

Now consider a standard monomial of the second type $w=r_{n-3} x_{n-1} v_{n}, n \geq 2$. In case $n=2 k, k \geq 1$, we have $r_{n-3}=x_{0}^{\xi_{0}} x_{1}^{\xi_{1}} \cdots x_{2 k-4}^{\xi_{2 k-4}} x_{2 k-3}^{\xi_{2 k-3}}, 0 \leq \xi_{i} \leq 1$, and $\operatorname{swt}\left(x_{2 k-1} v_{2 k}\right)=2$. We get

$$
\begin{gathered}
-k+1 \leq \operatorname{swt}\left(r_{n-3}\right) \leq k-1 ; \\
-\frac{n}{2}+3=-k+3 \leq \operatorname{swt}\left(r_{n-3} x_{n-1} v_{n}\right) \leq k+1=\frac{n}{2}+1 .
\end{gathered}
$$


Consider the case $n=2 k+1, k \geq 1$. Similarly, we get

$$
\begin{aligned}
-k & \leq \operatorname{swt}\left(r_{n-3}\right) \leq k-1 ; \\
-\frac{n}{2}-\frac{3}{2}=-k-2 & \leq \operatorname{swt}\left(r_{n-3} x_{n-1} v_{n}\right) \leq k-3=\frac{n}{2}-\frac{7}{2} .
\end{aligned}
$$

These two estimates yield the desired bounds for monomials of the second type.

We combine two previous lemmas along with a formal check in case $n=0$ for $\operatorname{swt}\left(v_{0}\right)=1$.

Lemma 7.3. Let $w$ be a standard monomial of length $n \geq 0$. Then

$$
\begin{aligned}
2^{n-2} & <\operatorname{wt}(w) \leq 2^{n}, \\
-\frac{n}{2}-\frac{3}{2} & \leq \operatorname{swt}(w) \leq \frac{n}{2}+1 .
\end{aligned}
$$

Theorem 7.4. The points of plane associated with the standard monomials of $\mathbf{R}=\operatorname{Lie}\left(v_{0}, v_{1}\right)$ are bounded by two logarithmic curves in terms of the weight coordinates $\mathrm{Wt}(w)=\left(Z_{1}, Z_{2}\right)$ :

$$
-\frac{1}{2} \log _{2} Z_{1}-\frac{5}{2}<Z_{2}<\frac{1}{2} \log _{2} Z_{1}+2 \text {. }
$$

Proof. Let $w$ be a standard monomial of length $n \geq 0$. By Lemma 7.3 , we have $2^{n-2}<\operatorname{wt}(w)=Z_{1}$, thus $n<\log _{2} Z_{1}+2$. By the same lemma, we get bounds on $Z_{2}=\operatorname{swt}(w)$ :

$$
-\frac{1}{2} \log _{2} Z_{1}-\frac{5}{2}<-\frac{n}{2}-\frac{3}{2} \leq Z_{2} \leq \frac{n}{2}+1<\frac{1}{2} \log _{2} Z_{1}+2 .
$$

\section{Structure of homogeneous components and growth of Lie superalgebra $\mathbf{R}$}

In this section we prove that the $\mathbb{Z}^{2}$-grading of $\mathbf{R}$ is fine (Theorem 8.4). We show that the growth of $\mathbf{R}=\operatorname{Lie}\left(v_{0}, v_{1}\right)$ is linear, moreover, we explicitly compute the weight growth function $\tilde{\gamma}_{\mathbf{R}}(m)$ and determine an asymptotic for the ordinary growth function $\gamma_{\mathbf{R}}(m)$ (Theorem 8.5).

First, we specify standard monomials of fixed weight.

Lemma 8.1. Suppose char $K \neq 2$ and fix an integer $m \geq 1$.

i) There is exactly one standard monomial of the first type $w_{1}$ with $\operatorname{wt}\left(w_{1}\right)=m$ (valid for char $K=2$ ).

ii) In the remaining claims assume that $m \notin\{1,3\}$. There is exactly one standard monomial of the second type $w_{2}$ with $\mathrm{wt}\left(w_{2}\right)=m$.

iii) There are exactly two standard monomials of weight $m$; (in case $m \in\{1,3\}$, there is exactly one standard monomial of weight $m$ ).

iv) Let $w_{1}=r_{n-2} v_{n}$ be as above, then $w_{2}=r_{n-2} x_{n} v_{n+1}$.

v) $\operatorname{swt}\left(w_{1}\right)-\operatorname{swt}\left(w_{2}\right)=3 \cdot(-1)^{n}$.

vi) $\operatorname{Gr}\left(w_{1}\right)-\operatorname{Gr}\left(w_{2}\right)=(-1)^{n}(2,-1)$.

Proof. We refine consideration of weights in Lemma 7.1. Fix $n \geq 1$. Consider all tails $r_{n-2}=x_{0}^{\xi_{0}} \cdots x_{n-2}^{\xi_{n-2}}$, $\xi_{i} \in\{0,1\}$, and respective weights wt $\left(r_{n-2}\right)=-\sum_{i=0}^{n-2} \xi_{i} 2^{i}$. By properties of the $p$-adic expansion of integers, there is a one-to-one correspondence between the set of these tails and the list of their weights, which fills an interval of integers $\left\{-2^{n-1}+1, \ldots,-1,0\right\}$ without gaps. Adding $\operatorname{wt}\left(v_{n}\right)=2^{n}$, we obtain a one-to-one correspondence between the set of standard monomials of the first type $r_{n-2} v_{n}$ and the list of their weights $\left\{2^{n-1}+1, \ldots, 2^{n}\right\}$, where $n \geq 1$ is fixed. We take unions of these sets and obtain a one-to-one correspondence between the set of standard monomials of the first type of length $n \geq 1$ and the list of their weights $m \geq 2$. It remains to consider the unique monomial of zero length $v_{0}, \operatorname{wt}\left(v_{0}\right)=1$. Claim (i) is proved.

Fix $n \geq 2$. By the arguments above, there is a one-to-one correspondence between the set of tails $r_{n-3}$ and the list of their weights $\left\{-2^{n-2}+1, \ldots,-1,0\right\}$ without gaps. Adding $\operatorname{wt}\left(x_{n-1} v_{n}\right)=-2^{n-1}+2^{n}=2^{n-1}$, we obtain a one-to-one correspondence between the set of standard monomials of the second type $r_{n-3} x_{n-1} v_{n}$ and the list of their weights $\left\{2^{n-2}+1, \ldots, 2^{n-1}\right\}$, where $n \geq 2$ is fixed. By taking unions of these sets, we obtain a one-to-one correspondence between the set of standard monomials of the second type of length $n \geq 2$ and their weights $m \geq 2$. It remains to exclude the false monomial of weight $\operatorname{wt}\left(x_{0} x_{2} v_{3}\right)=3$.

Claim (iii) follows from (i) and (ii).

To prove (iv), assume that $w_{1}=r_{n-2} v_{n}$ is a standard monomial of the first type with $\operatorname{wt}\left(w_{1}\right)=m \neq 1,3$. Clearly, $w_{2}=r_{n-2} x_{n} v_{n+1}$ is a standard monomial of the second type. It has the same weight $m$ :

$\operatorname{wt}\left(w_{2}\right)-\operatorname{wt}\left(w_{1}\right)=\operatorname{wt}\left(r_{n-2} x_{n} v_{n+1}\right)-\operatorname{wt}\left(r_{n-2} v_{n}\right)=\operatorname{wt}\left(x_{n}\right)+\operatorname{wt}\left(v_{n+1}\right)-\operatorname{wt}\left(v_{n}\right)=-2^{n}+2^{n+1}-2^{n}=0$. 
Using Lemma 6.1, we prove claim (v):

$$
\operatorname{swt}\left(w_{1}\right)-\operatorname{swt}\left(w_{2}\right)=\operatorname{swt}\left(r_{n-2} v_{n}\right)-\operatorname{swt}\left(r_{n-2} x_{n} v_{n+1}\right)=\operatorname{swt}\left(v_{n}\right)-\operatorname{swt}\left(x_{n}\right)-\operatorname{swt}\left(v_{n+1}\right)=3 \cdot(-1)^{n} .
$$

Finally, applying Lemma 6.6. we check claim (vi):

$$
\begin{aligned}
& \operatorname{Gr}\left(w_{1}\right)-\operatorname{Gr}\left(w_{2}\right)=\operatorname{Gr}\left(r_{n-2} v_{n}\right)-\operatorname{Gr}\left(r_{n-2} x_{n} v_{n+1}\right)=2 \operatorname{Gr}\left(v_{n}\right)-\operatorname{Gr}\left(v_{n+1}\right) \\
& =\frac{2}{3}\left(2^{n}+2(-1)^{n}, 2^{n}-(-1)^{n}\right)-\frac{1}{3}\left(2^{n+1}+2(-1)^{n+1}, 2^{n+1}-(-1)^{n+1}\right)=(-1)^{n}(2,-1) .
\end{aligned}
$$

Corollary 8.2. Fix $m \in \mathbb{N}, m \notin\{1,3\}$. Let $\left(X_{1}, X_{2}\right)$ and $\left(Z_{1}, Z_{2}\right)$ be the standard and weight coordinates on plane. The line of fixed weight $Z_{1}=X_{1}+2 X_{2}=m$ contains exactly two standard monomials whose position differ by vector $(2,-1)$. In case $m \in\{1,3\}$, the line contains one standard monomial. (see Fig. 11).

Corollary 8.3. Consider the weight $\mathbb{N}$-gradation $\mathbf{R}=\underset{n=1}{\oplus} \tilde{\mathbf{R}}_{n}$ (Section $\left[\right.$ G). Then $\operatorname{dim} \tilde{\mathbf{R}}_{n}=2$ for $n \geq 1$, except $\operatorname{dim} \tilde{\mathbf{R}}_{1}=\operatorname{dim} \tilde{\mathbf{R}}_{3}=1$.

Next, we easily derive that the multidegree $\mathbb{Z}^{2}$-gradation of $\mathbf{R}=\operatorname{Lie}\left(v_{0}, v_{1}\right)$ is fine.

Theorem 8.4. Components of the $\mathbb{Z}^{2}$-gradation $\mathbf{R}=\underset{n_{1}, n_{2} \geq 0}{\oplus} \mathbf{R}_{n_{1}, n_{2}}$ by multidegree in the generators $\left\{v_{0}, v_{1}\right\}$ (Lemma 6.3) are at most one-dimensional.

Proof. Consider a lattice point $\left(n_{1}, n_{2}\right) \in \mathbb{Z}^{2} \subset \mathbb{R}^{2}$. It belongs to a line of fixed weight $Z_{1}=X_{1}+2 X_{2}=$ $n_{1}+2 n_{2}$, that contains at most two standard monomials that differ by $(2,-1)$ (Corollary 8.2).

Theorem 8.5. The growth of the Lie superalgebra $\mathbf{R}$ is linear with the following properties.

i) Let char $K \neq 2$. We compute the weight growth function explicitly:

$$
\tilde{\gamma}_{\mathbf{R}}(m)=2 m-2, \quad m \geq 3 .
$$

ii) Let char $K=2$. We compute the weight growth function explicitly:

$$
\tilde{\gamma}_{\mathbf{R}}(m)=m+\left[\log _{2} m\right], \quad m \geq 1 .
$$

iii) $\operatorname{GKdim} \mathbf{R}=\underline{\mathrm{GK} \operatorname{dim}} \mathbf{R}=1$.

iv) Let char $K \neq 2$. The ordinary growth function satisfies: $\gamma_{\mathbf{R}}(m) \approx 3 m, m \rightarrow \infty$.

v) Let char $K=2$. The ordinary growth function satisfies: $\gamma_{\mathbf{R}}(m) \approx 3 m / 2, m \rightarrow \infty$.

Proof. Consider char $K \neq 2$. Using claim (iii) of Lemma 8.1 we compute the number of standard monomials of weight not exceeding $m$ and obtain that $\tilde{\gamma}_{\mathbf{R}}(m)=2 m-2, m \geq 3$.

Let char $K=2$. By Corollary 5.3 standard monomials of the first type and squares of the pivot elements, namely $\left\{x_{n-1} v_{n} \mid n \geq 2\right\}$, constitute a basis of $\mathbf{R}$. For each $m \geq 1$ we have exactly one standard monomial of the first type of weight $m$ (Lemma 8.1). One has $\operatorname{wt}\left(x_{n-1} v_{n}\right)=2^{n-1}, n \geq 2$. Now, claim (ii) follows. In any characteristic, we get GKdim $\mathbf{R}=\underline{\operatorname{GKdim}} \mathbf{R}=1$.

Let us prove claims (iv) and (v). Fix notations. Let $v$ be a standard monomial, $\left(X_{1}, X_{2}\right),\left(Z_{1}, Z_{2}\right)$ its multidegree and weight coordinates, and $n=\operatorname{deg}(v)=X_{1}+X_{2}$ its degree. Below, $m \in \mathbb{N}$ will be a parameter. The ordinary growth function $\gamma_{\mathbf{R}}(m), m \in \mathbb{N}$, counts standard monomials $v \in \mathbf{R}_{X_{1} X_{2}}$ of bounded degree, i.e. $n=X_{1}+X_{2} \leq m$. By (14), we have

$$
n=X_{1}+X_{2}=\frac{Z_{1}+2 Z_{2}}{3}+\frac{Z_{1}-Z_{2}}{3}=\frac{2}{3} Z_{1}+\frac{1}{3} Z_{2} .
$$

We apply estimates of Theorem 7.4

$$
\begin{aligned}
\frac{2}{3} Z_{1}-\frac{\log _{2} Z_{1}+5}{6} & \leq n \leq \frac{2}{3} Z_{1}+\frac{\log _{2} Z_{1}+4}{6} ; \\
\lim _{Z_{1} \rightarrow+\infty} \frac{n}{Z_{1}} & =\frac{2}{3} \\
\lim _{n \rightarrow+\infty} \frac{Z_{1}}{n} & =\frac{3}{2} .
\end{aligned}
$$

Hence, for any $\delta>0$ there exists $N_{\delta} \in \mathbb{N}$ such that

$$
\left(\frac{3}{2}-\delta\right) n \leq Z_{1} \leq\left(\frac{3}{2}+\delta\right) n, \quad n \geq N_{\delta} .
$$


Let $M_{\delta}$ be the number of standard monomials of degree less than $N_{\delta}$. Fix $m \in \mathbb{N}$. Consider a standard monomial $v$ of degree $n$ such that $N_{\delta} \leq n \leq m$. By upper bound (16),

$$
Z_{1} \leq\left(\frac{3}{2}+\delta\right) n \leq\left(\frac{3}{2}+\delta\right) m
$$

Now assume that char $K \neq 2$. For each weight $Z_{1}$ there are at most two standard monomials (Lemma 8.1). Using (17), we get an upper bound on the growth:

$$
\gamma_{\mathbf{R}}(m) \leq 2\left(\frac{3}{2}+\delta\right) m+M_{\delta}, \quad m \geq 1 .
$$

Consider standard monomials with bounded weight:

$$
Z_{1} \leq\left(\frac{3}{2}-\delta\right) m
$$

By lower inequality (16) it follows that $n \leq m$ for these standard monomials of degree $n \geq N_{\delta}$. For each weight $Z_{1}$ satisfying (19) we have two standard monomials (actually, we shall use (i)) and their degree is bounded by $m$. We also subtract the number $M_{\delta}$ of monomials which might not satisfy (16). So, we get a lower bound:

$$
\gamma_{\mathbf{R}}(m) \geq 2\left(\frac{3}{2}-\delta\right) m-2-M_{\delta}, \quad m \geq 1
$$

Since $\delta>0$ was chosen arbitrary, (18) and (20) imply that $\lim _{m \rightarrow \infty} \gamma_{\mathbf{R}}(m) / m=3$.

Consider char $K=2$. Similarly, using item (ii) of Theorem 8.5 we get

$$
\left(\frac{3}{2}-\delta\right) m-M_{\delta} \leq \gamma_{\mathbf{R}}(m) \leq\left(\frac{3}{2}+\delta\right) m+\left[\log _{2}((3 / 2+\delta) m)\right]+M_{\delta}, \quad m \geq 1 .
$$

We conclude that $\lim _{m \rightarrow \infty} \gamma_{\mathbf{R}}(m) / m=3 / 2$.

\section{Basis, bounds on weights, and growth of associative hull A}

Now our goal is to determine growth of the associative hull $\mathbf{A}=\operatorname{Alg}\left(v_{0}, v_{1}\right)$. For a series of previous examples of (fractal, or self-similar) (restricted) Lie (super)algebras, bases for respective associative hulls were not found 44, 48, 46, 42, 41. Instead, we used a bypass approach. We considered bigger (restricted) Lie (super)algebras $\tilde{\mathbf{R}} \supset \mathbf{R}$ whose bases were given by so called quasi-standard monomials and we determined and used bases of their associative hulls $\tilde{\mathbf{A}}=\operatorname{Alg}(\tilde{\mathbf{R}}) \supset \mathbf{A}$.

The virtue of the present example is that we are able to describe explicitly a basis of the associative hull $\mathbf{A}$. In this section we find a basis of $\mathbf{A}$ and establish bounds on its weights. These bounds allow us to describe a geometric position of the basis of $\mathbf{A}$ on plane. Finally, we specify the growth of $\mathbf{A}$.

Theorem 9.1. Let $\mathbf{A}=\operatorname{Alg}\left(v_{0}, v_{1}\right) \subset \operatorname{End}(\Lambda)$, char $K \neq 2$. Then

i) a basis of $\mathbf{A}$ is given by monomials

$$
\begin{aligned}
& \left\{v_{0}, v_{1} v_{0}^{*}\right\} \bigcup\left\{x_{0}^{\xi_{0}} x_{1}^{\xi_{1}} v_{2} v_{1}^{*} v_{0}^{*} \mid \xi_{0}+\xi_{1} \leq 1\right\} \bigcup \\
& \bigcup_{n=3}^{\infty}\left\{r_{n-3} x_{n-2}^{\xi_{n-2}} x_{n-1}^{\xi_{n-1}} v_{n} v_{n-1}^{\alpha_{n-1}} v_{n-2}^{\alpha_{n-2}} \cdots v_{0}^{\alpha_{0}} \mid \xi_{i}, \alpha_{i} \in\{0,1\} ; \xi_{n-2}+\xi_{n-1} \leq 1+\alpha_{n-1}\right\},
\end{aligned}
$$

where $r_{n-3}$ are tail monomials, and $b^{*}$ denote all $b^{\alpha}, \alpha \in\{0,1\}$. In case $n=3$, we additionally assume that the monomials containing both $\left\{x_{0}, x_{2}\right\}$ are only of type: $x_{0} x_{2} v_{3} v_{2} v_{1}^{*} v_{0}^{*}$;

ii) let char $K=2$, then $\mathbf{A}$ is contained in span of the monomials above;

iii) monomials with $\xi_{n-1}=0$ are linearly independent and belong to $\mathbf{A}$ in case of any field.

Proof. We refer to $n$ above as to the length of the respective monomial. First, we check that the listed monomials belong to A. Let $n \geq 4$. Consider $w=r_{n-3} x_{n-2}^{\xi_{n-2}} x_{n-1}^{\xi_{n-1}} v_{n} v_{n-1}^{\alpha_{n-1}} v_{n-2}^{\alpha_{n-2}} \cdots v_{0}^{\alpha_{0}}$, satisfying our condition $\xi_{n-2}+\xi_{n-1} \leq 1+\alpha_{n-1}$. We have two cases a) If $\xi_{n-2}+\xi_{n-1} \leq 1$, then the initial factor $r_{n-3} x_{n-2}^{\xi_{n-2}} x_{n-1}^{\xi_{n-1}} v_{n}$ is a standard monomial. Since $v_{i} \in \mathbf{R}$, we conclude that $w \in \mathbf{A}$. b) Consider the case $\xi_{n-2}=\xi_{n-1}=1$, by the assumed inequality, $\alpha_{n-1}=1$. Then also

$$
w=\left(r_{n-3} x_{n-1} v_{n}\right) \cdot\left(x_{n-2} v_{n-1}\right) \cdot v_{n-2}^{\alpha_{n-2}} \cdots v_{0}^{\alpha_{0}} \in \mathbf{A} .
$$

The cases $n=0,1,2$ are easily checked. Consider $n=3$. The arguments in two cases above fail only when they lead to using the false monomial $x_{0} x_{2} v_{3}$ as the initial factor. Thus, the algorithm above fail to get monomials containing both $\left\{x_{0}, x_{2}\right\}$. We have to check that we can obtain the monomials specified in the 
wording that contain both $\left\{x_{0}, x_{2}\right\}$. To obtain the claimed monomials we use that $x_{0} x_{2} v_{3} v_{2}=x_{2} v_{3} \cdot x_{0} v_{2} \in \mathbf{A}$. The linear independence follows by the same arguments as [44, proof of Theorem 4.1].

Second, we shall prove that products of the standard monomials are expressed via the associative monomials listed above. We work with products of standard monomials, we reorder such products using PBW-like arguments, where a total order is fixed obeying to the length $n$ of standard monomials. In this process, we shall eliminate not only squares of odd standard monomials but also products of two standard monomials of the same length:

$$
\left(r_{n-1} v_{n}\right) \cdot\left(r_{n-1}^{\prime} v_{n}\right)= \pm r_{n-1}^{\prime \prime} v_{n}^{2}= \pm r_{n-1}^{\prime \prime} x_{n+1} v_{n+2} .
$$

The obtained monomial is standard (we cannot obtain the false monomial because one has the only unsuccessful possibility to get it: $\left.v_{1} \cdot v_{1}=x_{2} v_{3}\right)$. As a result, we get products of standard monomials of different lengths, written in strictly length-decreasing order. For example, we obtain:

$$
r_{n-1} v_{n} \cdot\left(r_{n-2} v_{n-1}\right) \cdots\left(v_{0}\right),
$$

where we have one standard monomial of length $n$, while the remaining standard monomials of lengths $n-1, \ldots, 0$ are optional.

Consider $n \geq 4$. Now, we move all Grassmann letters in (21) to the left. Let $x_{i}$ be a Grassmann variable in a standard monomial $r_{j-1} v_{j}, j<n$, then $i<j$. The standard monomials before it in (21) have lengths greater than $j$, thus, greater than $i$. So, $x_{i}$ supercommutes with all preceding heads $v_{k}$. We obtain an associative monomial as desired, we only need to check the inequality on the senior indices. Consider two cases. a) The factor $r_{n-2} v_{n-1}$ does not appear in (21). The senior factor $r_{n-1} v_{n}$ is standard, thus, it contains at most one of the variables $\left\{x_{n-2}, x_{n-1}\right\}$. Since it is not possible to get any of these variables from monomials of smaller length, we get $\xi_{n-2}+\xi_{n-1} \leq 1$ and $\alpha_{n-1}=0$, and the required inequality is valid. b) The factor $r_{n-2} v_{n-1}$ appears in (21). Then we get $\alpha_{n-1}=1$ and the desired inequality on the senior indices is satisfied.

Consider $n=2$. Recall that all factors in (21) are standard monomials. So, the first factor is either $x_{0} v_{2}$ or $x_{1} v_{2}$, followed by optional factors $v_{1}, v_{0}$. We get the desired monomials of length 2 . The cases $n=0,1$ are trivial.

Consider $n=3$. We can get $x_{2}$ in (21) only from the first factor. By Theorem 5.1, there is only one such standard monomial, namely, $r_{2} v_{3}=x_{2} v_{3}$. Now, $x_{0}$ can appear only from the second factor, and there is only one such standard monomial: $r_{1} v_{2}=x_{0} v_{2}$. The possible remaining factors are $v_{1}$ and $v_{0}$. Thus, we arrive at the claimed monomials: $x_{0} x_{2} v_{3} v_{2} v_{1}^{*} v_{0}^{*}$. The first claim is proved.

The second claim follows by the same arguments, some monomials can only disappear. The third claim follows because $r_{n-2} v_{n}, v_{n-1}, \ldots, v_{0} \in \mathbf{R} \subset \mathbf{A}$.

Lemma 9.2. Let $w=r_{n-3} x_{n-2}^{\xi_{n-2}} x_{n-1}^{\xi_{n-1}} v_{n} v_{n-1}^{\alpha_{n-1}} v_{n-2}^{\alpha_{n-2}} \cdots v_{0}^{\alpha_{0}}$ be a basis monomial of $\mathbf{A}$ of length $n$. Then

$$
2^{n-2}<\operatorname{wt}(w)<2^{n+1}, \quad|\operatorname{swt}(w)| \leq n+1 ; \quad n \geq 0 .
$$

Proof. For $n \in\{0,1,2\}$, these estimates are verified directly. Suppose that $n \geq 3$. Let $w$ contain at most one of Grassmann variables $\left\{x_{n-2}, x_{n-1}\right\}$, we have

$$
2^{n-2}<2^{n-2}+1=-\left(2^{0}+\cdots+2^{n-3}\right)-2^{n-1}+2^{n} \leq \operatorname{wt}(w) \leq 2^{n}+2^{n-1}+\cdots+2^{0}=2^{n+1}-1<2^{n+1} .
$$

In case $\xi_{n-2}=\xi_{n-1}=1, w$ must contain the factor $v_{n-1}$. So, we have the desired estimates again:

$$
2^{n-2}<2^{n-1}+1=-\left(2^{0}+\cdots+2^{n-1}\right)+2^{n}+2^{n-1} \leq \operatorname{wt}(w) \leq 2^{n}+2^{n-1}+\cdots+2^{0}=2^{n+1}-1<2^{n+1} .
$$

Consider the superweight. Let $n=2 k$. By estimates $-k \leq \operatorname{swt}\left(r_{n-1}\right) \leq k, \operatorname{swt}\left(v_{2 k}\right)=1$, and $-k \leq$ $\operatorname{swt}\left(v_{2 k-1}^{\alpha_{2 k-1}} \cdots v_{0}^{\alpha_{0}}\right) \leq k$, we have

$$
-2 k+1 \leq \operatorname{swt}(w) \leq 2 k+1 .
$$

Let $n=2 k+1$. We have $-k-1 \leq \operatorname{swt}\left(r_{n-1}\right) \leq k, \operatorname{swt}\left(v_{2 k+1}\right)=-1,-k \leq \operatorname{swt}\left(v_{2 k}^{\alpha_{2 k}} \cdots v_{0}^{\alpha_{0}}\right) \leq k+1$, thus,

$$
-2 k-2 \leq \operatorname{swt}(w) \leq 2 k \text {. }
$$

Both cases yield $|\operatorname{swt}(w)| \leq n+1$.

Theorem 9.3. Points of plane depicting the basis monomials of $\mathbf{A}=\operatorname{Alg}\left(v_{0}, v_{1}\right)$ are bounded by two logarithmic curves in terms of the weight coordinates $\mathrm{Wt}(w)=\left(Z_{1}, Z_{2}\right)$ :

$$
\left|Z_{2}\right|<\log _{2} Z_{1}+3 \text {. }
$$

Proof. Let $w$ be a basis monomial of $\mathbf{A}$ of length $n$. By Lemma 9.2 one has estimates: $2^{n-2}<\operatorname{wt}(w)=Z_{1}$, and $\left|Z_{2}\right|=|\operatorname{swt}(w)| \leq n+1$. Thus, $\left|Z_{2}\right|<\log _{2} Z_{1}+3$.

Theorem 9.4. Let $\mathbf{A}=\operatorname{Alg}\left(v_{0}, v_{1}\right)$. The growth of $\mathbf{A}$ is quadratic, namely: 
i) there exist constants $c_{1}, c_{2}, c_{3}, c_{4}>0$ such that

(a) $c_{1} m^{2} \leq \tilde{\gamma}_{\mathbf{A}}(m) \leq c_{2} m^{2}, m \geq 1$ (the weight growth function);

(b) $c_{3} m^{2} \leq \gamma_{\mathbf{A}}(m) \leq c_{4} m^{2}, m \geq 1$ (the ordinary growth function);

ii) $\operatorname{GKdim} \mathbf{A}=\underline{G K d i m} \mathbf{A}=2$.

Proof. First, let us establish bounds on the weight growth function. Let $m \geq 2$ be fixed, set $n=\left[\log _{2} m\right]-$ 1. Consider all monomials $w=r_{n-2} v_{n} v_{n-1}^{\alpha_{n-1}} v_{n-2}^{\alpha_{n-2}} \cdots v_{0}^{\alpha_{0}} \in \mathbf{A}$ (i.e. $\xi_{n-1}=0$, Theorem 9.1, (iii)). By Lemma 9.2, $\operatorname{wt}(w)<2^{n+1} \leq m$. The number of these monomials yields a desired lower bound on the weight growth function:

$$
\tilde{\gamma}_{\mathbf{A}}(m) \geq 2^{n-1} \cdot 2^{n}=2^{2 n-1} \geq 2^{2 \log _{2} m-5}=\frac{1}{32} m^{2} .
$$

Fix $m \geq 1$ and set $n=\left[\log _{2} m\right]+3$. Consider a monomial $w=r_{j-3} x_{j-2}^{\xi_{j-2}} x_{j-1}^{\xi_{j-1}} v_{j} v_{j-1}^{\alpha_{j-1}} \cdots v_{0}^{\alpha_{0}} \in \mathbf{A}$ (without inequality on indices of Theorem 9.1). If $j>n$, then using Lemma $9.2 \mathrm{wt}(w)>2^{j-2}>2^{n-2}>m$. Thus, consider monomials $w$ of bounded weight: $\operatorname{wt}(w) \leq m$, then for all of them we have $j \leq n$. Therefore, the number of monomials $w$ of length at most $n$ yields an upper bound on the weight growth function $\tilde{\gamma}_{\mathbf{A}}(m)$. Let $j \geq 0$. We have $2^{j}$ possibilities for the Grassmann variables and $2^{j}$ possibilities for $\alpha_{j-1}, \ldots, \alpha_{0}$. So, we get a desired upper bound on the weight growth function:

$$
\tilde{\gamma}_{\mathbf{A}}(m) \leq \sum_{j=0}^{n} 2^{2 j}<\frac{4^{n+1}}{3} \leq \frac{4^{\log _{2} m+4}}{3}=\frac{2^{8}}{3} m^{2} .
$$

We have $1 \leq \operatorname{wt}\left(v_{0}\right), \operatorname{wt}\left(v_{1}\right) \leq 2$. One observes that $\tilde{\gamma}_{\mathbf{A}}(m) \leq \gamma_{\mathbf{A}}(m) \leq \tilde{\gamma}_{\mathbf{A}}(2 m), m \geq 1$. Now one obtains bounds on the ordinary growth function. Part (ii) is trivial.

Remark 1. Probably, one can find the weight growth function of $\mathbf{A}$ explicitly similar to Theorem 8.5 .

\section{Generating Functions of Lie superalgebra $\mathbf{R}$}

Recall that components of the $\mathbb{Z}^{2}$-gradation of $\mathbf{R}$ are at most one-dimensional (Theorem 8.4). In this section we study this gradation in terms of generating functions. We establish recursive relations. The recursive relations allows us to compute an initial part of the generating function, which simplifies the proof that $\mathbf{R}$ is of finite width in the next section.

Let $A=\underset{n, n \in \mathbb{Z}}{\bigoplus} A_{n m}$ be a $\mathbb{Z}^{2}$-graded algebra and $A=\underset{n \in \mathbb{Z}}{\oplus} A_{n}$, where $A_{n}=\underset{m+k=n}{\oplus} A_{m k}$, a induced $\mathbb{Z}$-gradation. Define respective generating functions (or Hilbert functions):

$$
\begin{aligned}
\mathcal{H}\left(A, t_{1}, t_{2}\right) & =\sum_{n, m} \operatorname{dim} A_{n m} t_{1}^{n} t_{2}^{m} ; \\
\mathcal{H}(A, t) & =\sum_{n} \operatorname{dim} A_{n} t^{n}=\mathcal{H}(A, t, t) .
\end{aligned}
$$

Similarly, we define generating functions for graded sets and spaces. Recall that the Lie superalgebra $\mathbf{R}=\operatorname{Lie}\left(v_{0}, v_{1}\right)$ is $\mathbb{Z}^{2}$-graded by multidegree in the generators (Lemma 6.3). Denote by $T_{n}^{k}$ the set of standard monomials of length $n$ and type $k$, by $T_{n}$ all standard monomials of length $n$, where $n \geq 0$, $k \in\{1,2\}$. Let $T$ be the set of all standard monomials, it is a homogeneous basis of $\mathbf{R}=\operatorname{Lie}\left(v_{0}, v_{1}\right)$ (in case char $K \neq 2$ ).

Lemma 10.1. Let $U \subset T$ be a subset of standard monomials and $\tau: \mathbf{R} \rightarrow \mathbf{R}$ the shift endomorphism. Then

$$
\mathcal{H}\left(\tau(U), t_{1}, t_{2}\right)=\mathcal{H}\left(U, t_{2}, t_{1}^{2} t_{2}\right)
$$

Proof. Consider $a \in U_{n_{1}, n_{2}}$. By Lemma 6.8 $\operatorname{Gr}(\tau(a))=\left(2 n_{2}, n_{1}+n_{2}\right)$. Hence,

$$
\mathcal{H}\left(\tau\left(U_{n_{1}, n_{2}}\right), t_{1}, t_{2}\right)=\operatorname{dim} U_{n_{1}, n_{2}} t_{1}^{2 n_{2}} t_{2}^{n_{1}+n_{2}}=\operatorname{dim} U_{n_{1}, n_{2}} t_{2}^{n_{1}}\left(t_{1}^{2} t_{2}\right)^{n_{2}}=\mathcal{H}\left(U_{n_{1}, n_{2}}, t_{2}, t_{1}^{2} t_{2}\right) .
$$

Lemma 10.2. Let char $K \neq 2$. For all lengths $n \geq 4$ we have bijections:

i) $T_{n+1}^{k}=\left\{1, x_{0}\right\} \cdot \tau\left(T_{n}^{k}\right)$ for $k \in\{0,1\}$, (in case $k=1$, valid also for $n \geq 1$ );

ii) $T_{n+1}=\left\{1, x_{0}\right\} \cdot \tau\left(T_{n}\right)$;

iii) and equalities: $\mathcal{H}\left(T_{n+1}, t_{1}, t_{2}\right)=\left(1+t_{1}^{-1}\right) \mathcal{H}\left(\tau\left(T_{n}\right), t_{1}, t_{2}\right)=\left(1+t_{1}^{-1}\right) \mathcal{H}\left(T_{n}, t_{2}, t_{1}^{2} t_{2}\right)$.

Proof. The first and second claim follow directly by the structure of the standard monomials (Theorem 5.1). Since $\operatorname{Gr}\left(x_{0}\right)=-\operatorname{Gr}\left(v_{0}\right)=(-1,0)$, we get $\mathcal{H}\left(\left\{1, x_{0}\right\}, t_{1}, t_{2}\right)=1+t_{1}^{-1}$. It remains to apply Lemma 10.1. 
FiguRE 1. One-dimensional components $\mathbf{R}_{n, m}$ are shown by dots. Green and blue points are standard monomials of the first and second types, respectively. Dashed red arrows point to pivot elements and red lines depict points with weights equal to that of pivot elements. Two green logarithmic curves restrict positions of standard monomials. Grey dashed lines show points with constant degrees.

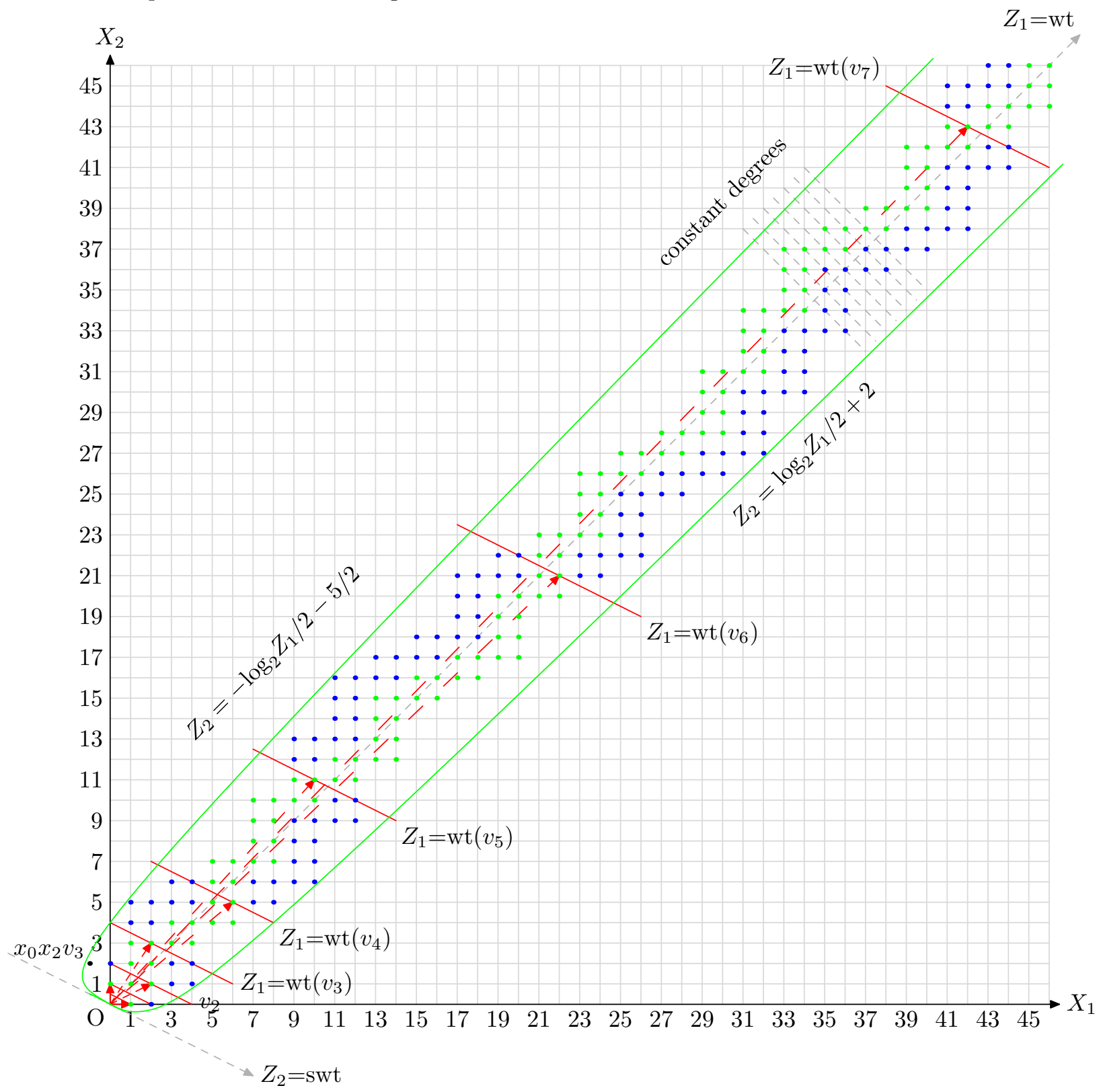

Lemma 10.3. Let $\mathbf{R}=\operatorname{Lie}\left(v_{0}, v_{1}\right)$, char $K \neq 2$. Then $\mathcal{H}\left(\mathbf{R}, t_{1}, t_{2}\right)$ satisfies a recursive relation:

$$
\mathcal{H}\left(\mathbf{R}, t_{1}, t_{2}\right)=t_{1}+t_{1}^{2}+t_{1}^{3} t_{2}+t_{1}^{4} t_{2}-t_{1}^{-1} t_{2}-t_{1}^{-1} t_{2}^{2}+\left(1+t_{1}^{-1}\right) \cdot \mathcal{H}\left(\mathbf{R}, t_{2}, t_{1}^{2} t_{2}\right) .
$$

Proof. We have

$$
\begin{aligned}
& T_{0}=\left\{v_{0}\right\} ; \quad T_{1}=\left\{v_{1}\right\} ; \quad T_{2}=\left\{v_{2}, x_{0} v_{2}, x_{1} v_{2}\right\} ; \\
& T_{3}=\left\{v_{3}, x_{0} v_{3}, x_{1} v_{3}, x_{2} v_{3}, x_{0} x_{1} v_{3}\right\} ; \\
& T_{4}=\left\{v_{4}, x_{0} v_{4}, x_{1} v_{4}, x_{2} v_{4}, x_{3} v_{4}, x_{0} x_{1} v_{4}, x_{0} x_{2} v_{4}, x_{0} x_{3} v_{4}, x_{1} x_{2} v_{4}, x_{1} x_{3} v_{4}, x_{0} x_{1} x_{2} v_{4}, x_{0} x_{1} x_{3} v_{4}\right\} .
\end{aligned}
$$


We find the respective generating functions:

$$
\begin{aligned}
& \mathcal{H}\left(T_{0}, t_{1}, t_{2}\right)=t_{1} ; \quad \mathcal{H}\left(T_{1}, t_{1}, t_{2}\right)=t_{2} ; \quad \mathcal{H}\left(T_{2}, t_{1}, t_{2}\right)=t_{1}^{2}+t_{1} t_{2}+t_{1}^{2} t_{2} ; \\
& \mathcal{H}\left(T_{3}, t_{1}, t_{2}\right)=t_{2}^{2}+t_{1} t_{2}^{2}+t_{1}^{2} t_{2}^{2}+t_{1} t_{2}^{3}+t_{1}^{2} t_{2}^{3} ; \\
& \mathcal{H}\left(T_{4}, t_{1}, t_{2}\right)=t_{1}^{3} t_{2}+t_{1}^{4} t_{2}+t_{1}^{3} t_{2}^{2}+t_{1}^{4} t_{2}^{2}+t_{1}^{3} t_{2}^{3}+t_{1}^{4} t_{2}^{3}+t_{1}^{3} t_{2}^{4}+t_{1}^{4} t_{2}^{4}+t_{1}^{5} t_{2}^{4}+t_{1}^{6} t_{2}^{4}+t_{1}^{5} t_{2}^{5}+t_{1}^{6} t_{2}^{5}
\end{aligned}
$$

By Lemma 10.2, we have the following bijection:

$$
T \backslash\left(T_{0} \cup T_{1} \cup T_{2} \cup T_{3} \cup T_{4}\right)=\left\{1, x_{0}\right\} \cdot \tau\left(T \backslash\left(T_{0} \cup T_{1} \cup T_{2} \cup T_{3}\right)\right) .
$$

Using (22) and Lemma 10.1, we establish the claimed relation:

$$
\begin{aligned}
\mathcal{H}\left(\mathbf{R}, t_{1}, t_{2}\right)= & \mathcal{H}\left(T_{0} \cup T_{1} \cup T_{2} \cup T_{3} \cup T_{4}, t_{1}, t_{2}\right)+\left(1+t_{1}^{-1}\right) \cdot \mathcal{H}\left(\tau\left(T \backslash\left(T_{0} \cup T_{1} \cup T_{2} \cup T_{3}\right)\right), t_{1}, t_{2}\right) \\
= & \mathcal{H}\left(T_{0} \cup T_{1} \cup T_{2} \cup T_{3} \cup T_{4}, t_{1}, t_{2}\right) \\
& +\left.\left(1+t_{1}^{-1}\right) \cdot\left(\mathcal{H}\left(\mathbf{R}, t_{1}, t_{2}\right)-\mathcal{H}\left(T_{0} \cup T_{1} \cup T_{2} \cup T_{3}, t_{1}, t_{2}\right)\right)\right|_{t_{1}:=t_{2}, t_{2}:=t_{1}^{2} t_{2}} \\
= & t_{1}+t_{2}+t_{1}^{2}+t_{1} t_{2}+t_{2}^{2}+t_{1}^{2} t_{2}+t_{1} t_{2}^{2}+t_{1}^{3} t_{2}+t_{1}^{2} t_{2}^{2}+t_{1} t_{2}^{3}+t_{1}^{4} t_{2}+t_{1}^{3} t_{2}^{2} \\
& +t_{1}^{2} t_{2}^{3}+t_{1}^{4} t_{2}^{2}+t_{1}^{3} t_{2}^{3}+t_{1}^{4} t_{2}^{3}+t_{1}^{3} t_{2}^{4}+t_{1}^{4} t_{2}^{4}+t_{1}^{5} t_{2}^{4}+t_{1}^{6} t_{2}^{4}+t_{1}^{5} t_{2}^{5}+t_{1}^{6} t_{2}^{5} \\
& +\left(1+t_{1}^{-1}\right) \cdot \mathcal{H}\left(\mathbf{R}, t_{2}, t_{1}^{2} t_{2}\right)-t_{2}-t_{1}^{2} t_{2}-t_{1}^{2} t_{2}^{2}-t_{2}^{2}-t_{1}^{2} t_{2}^{3}-t_{1}^{4} t_{2}^{2}-t_{1}^{4} t_{2}^{4} \\
& -t_{1}^{6} t_{2}^{5}-t_{1}^{6} t_{2}^{4}-t_{1}^{4} t_{2}^{3}-t_{1}^{-1} t_{2}-t_{1} t_{2}-t_{1} t_{2}^{2}-t_{1}^{-1} t_{2}^{2}-t_{1} t_{2}^{3}-t_{1}^{3} t_{2}^{2}-t_{1}^{3} t_{2}^{4}-t_{1}^{5} t_{2}^{5}-t_{1}^{5} t_{2}^{4}-t_{1}^{3} t_{2}^{3} \\
= & t_{1}+t_{1}^{2}+t_{1}^{3} t_{2}+t_{1}^{4} t_{2}-t_{1}^{-1} t_{2}-t_{1}^{-1} t_{2}^{2}+\left(1+t_{1}^{-1}\right) \cdot \mathcal{H}\left(\mathbf{R}, t_{2}, t_{1}^{2} t_{2}\right) .
\end{aligned}
$$

Remark 2. On Fig. 1 all diagonal components are occupied. But in general, this observation is not true. We give a list of indices of initial empty diagonal components $n$ (i.e. $\operatorname{dim} \mathbf{R}_{n, n}=0$ ):

$57,114,185,217,225,227,228,229,233,249,313,370,434,450,454,455,456,458,466,498,569,626,697$,

$729,737,739,740,741,745,761,825,857,865,867,868,869,873,889,897,899,900,901,905,907,908$,

$909,910,911,912,913,915,916,917,921,929,931,932,933,937,953,985,993,995,996,997,1001, \ldots$

\section{Finite width of Lie superalgebra R}

Let $\mathbf{R}=\operatorname{Lie}\left(v_{0}, v_{1}\right) \subset$ Der $\Lambda$ be the Lie superalgebra as above. Consider the degree (natural) $\mathbb{N}$-gradation

$\mathbf{R}=\underset{n=1}{\infty} \mathbf{R}_{n}$, where $\mathbf{R}_{n}$ is spanned by basis elements of degree $n$ in the generators $\left\{v_{0}, v_{1}\right\}, n \geq 1$. Consider the respective generating function $\mathcal{H}(\mathbf{R}, t)=\sum_{n=1}^{\infty} \operatorname{dim} \mathbf{R}_{n} t^{n}$. In case char $K \neq 2$, a computer computation based on Lemma 10.2 yields that all coefficients are $\{2,3,4\}$, this was checked till degree 4000 :

$$
\begin{aligned}
& \mathcal{H}(\mathbf{R}, t)=2 t+3 t^{2}+2 t^{3}+3 t^{4}+4 t^{5}+4 t^{6}+3 t^{7}+2 t^{8}+3 t^{9}+3 t^{10}+2 t^{11}+3 t^{12}+3 t^{13}+2 t^{14}+3 t^{15} \\
& \quad+4 t^{16}+4 t^{17}+3 t^{18}+2 t^{19}+3 t^{20}+4 t^{21}+4 t^{22}+3 t^{23}+2 t^{24}+3 t^{25}+4 t^{26}+4 t^{27}+3 t^{28}+2 t^{29}+3 t^{30} \\
& \quad+3 t^{31}+2 t^{32}+3 t^{33}+3 t^{34}+2 t^{35}+3 t^{36}+4 t^{37}+4 t^{38}+3 t^{39}+2 t^{40}+3 t^{41}+3 t^{42}+2 t^{43}+3 t^{44}+3 t^{45} \\
& \quad+2 t^{46}+3 t^{47}+4 t^{48}+4 t^{49}+3 t^{50}+2 t^{51}+3 t^{52}+3 t^{53}+2 t^{54}+3 t^{55}+3 t^{56}+2 t^{57}+3 t^{58}+4 t^{59}+4 t^{60}+\ldots
\end{aligned}
$$

Let us prove that this observation is true in general.

Theorem 11.1. Let $\mathbf{R} \subset$ Der $\Lambda$ be an algebra of type specified below generated by $\left\{v_{0}, v_{1}\right\}$ over a field $K$, $\mathbf{R}=\bigoplus_{n=1}^{\infty} \mathbf{R}_{n}$ the degree $\mathbb{N}$-gradation, and $\left(\mathbf{R}^{n} \mid n \geq 1\right)$ the lower central series. By Lemma 6.9, we have $a_{n}=\operatorname{dim} \mathbf{R}_{n}=\operatorname{dim} \mathbf{R}^{n} / \mathbf{R}^{n+1}, n \geq 1$. Then

i) Let char $K \neq 2, \mathbf{R}=\operatorname{Lie}\left(v_{0}, v_{1}\right)$ the Lie superalgebra. Then $a_{n} \in\{2,3,4\}$, and $\mathbf{R}$ is of width 4 .

ii) Let char $K=2$, $\mathbf{R}$ the Lie algebra. Then $a_{n} \in\{1,2\}$, and $\mathbf{R}$ is of width 2.

iii) Let char $K=2$, $\mathbf{R}$ the Lie superalgebra (or the restricted Lie (super)algebra). Then $a_{n} \in\{1,2,3\}$, and $\mathbf{R}$ is of width 3.

Proof. We start with the second claim. By Corollary 5.3 , a basis of $\mathbf{R}$ is given by the standard monomials of the first type. On Fig. 2 these monomials are depicted by green points except the pivot elements which are red. By Lemma 8.1 for any $m \geq 1$ there is exactly one monomial of the first type and weight $m$. By Lemma 7.1 all monomials $w \in T_{n}^{1}$ are between two parallel red lines of fixed weight, namely, $2^{n-1}<\operatorname{wt}(w) \leq 2^{n}, n \geq 0$. Thus, the sets $T_{n}^{1}, n \geq 0$, are in different regions of plane separated by these red lines. We connect $T_{n}^{1}$, the 
Figure 2. Notations are the same as on Fig. 1

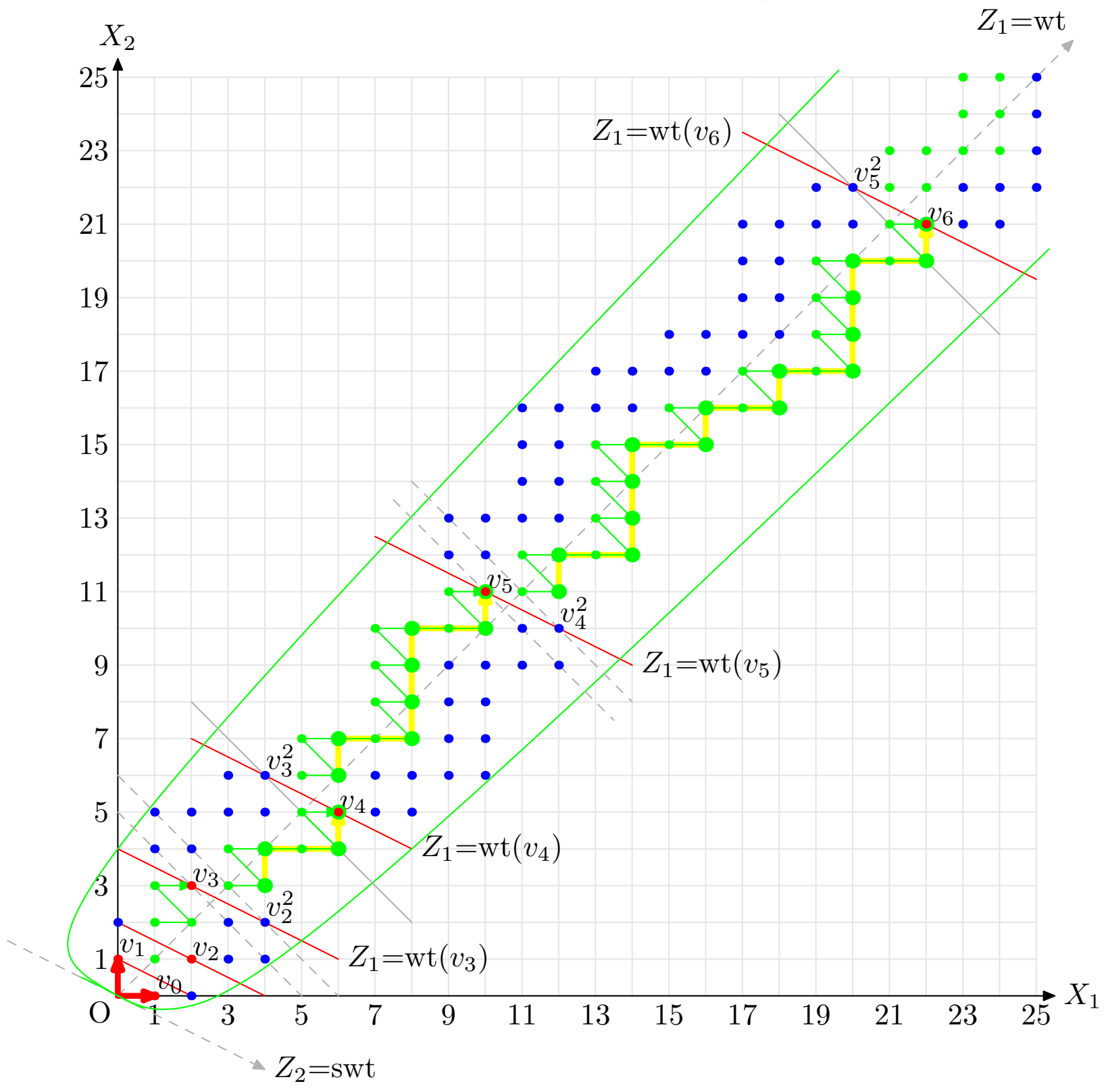

set of the standard monomials of the first type of length $n$, where $n \geq 3$, by a thin green broken line in order of their weights. We prove and use the following geometric phenomena labeled by letters.

(A) The broken line for $T_{n}^{1}, n \geq 3$, consists of alternating parts of two types, namely, an oblique segment $a=(-1,1)$ is followed by either a horizontal segment $b=(1,0)$ or the three horizontal segments $b$. One checks that the steps $a$ and $b$, indeed, increase weights of vertices by one. We prove (A) by induction on $n$. The green broken lines for $T_{n}^{1}, 3 \leq n \leq 6$, are shown on Fig. 2, they clearly satisfy (A). The case $n=3$ is the base of the induction. Arguing by induction, assume that the monomials of $T_{n}^{1}$, where $n \geq 3$ is fixed, satisfy (A). By Lemma 10.2 we have the bijection $T_{n+1}^{1}=\left\{1, x_{0}\right\} \cdot \tau\left(T_{n}^{1}\right)$. Since $\tau$ acts on plane as a liner transformation described in Lemma 6.8 the application of $\tau$ produces a new broken line consisting of new alternating parts: a horizontal long segment $\tilde{a}=\tau(a)=(2,0)$ followed by either a vertical segment $\tilde{b}=\tau(b)=(0,1)$ or the three vertical segments $\tilde{b}$. These new broken lines are drawn on Fig. 2 using thick yellows segments. Now we take into account the action of two components of the factor $\left\{1, x_{0}\right\}$. We conclude that $T_{n+1}^{1}$ consists of the vertices of the new broken yellow line, shown as thick points, and their horizontal shifts by $\operatorname{Gr}\left(x_{0}\right)=(-1,0)$. Now one really requires geometric observations, e.g. drawing the process on a 
checkered paper or scrutinize it on Fig. 2 to observe that we get points belonging to a new broken green line as required, thus proving the inductive step.

(B) A transformation of the broken line in the process above: $T_{n}^{1} \rightsquigarrow T_{n+1}^{1}, n \geq 3$, is described by a replacement rule $\tilde{\tau}: a \mapsto b b, b \mapsto a b$, concatenating a prefix $b$ to the result. One needs to repeat and refine the geometric observations above. (The replacement rule is also formally valid for $n=1,2$ ).

(C) The broken lines for $T_{n}^{1}, n \geq 4$, keep the same initial and final segments: babbba...abbbab (may be, with overlapping). The cases $n=4,5,6$ are seen on Fig. 2. This property follows from (B).

(D) We claim that $T_{n-1}^{1}$ and $T_{n}^{1}$, where $n \geq 1$, are connected either by $b$ or a, for even and odd $n$, respectively. For $n=1$ the claim is trivial. Let $n \geq 2$. The broken line for $T_{n-1}^{1}$ ends with $v_{n-1}$, while one for $T_{n}^{1}$ starts with $w=x_{0} \cdots x_{n-2} v_{n}$, because this is the element in $T_{n}^{1}$ of smallest weight. Assume that $n$ is even, by Lemma 4.2. $\left[v_{0}, v_{n-1}\right]=-x_{0} \cdots x_{n-2} v_{n}=-w$. We get $\operatorname{Wt}(w)=\operatorname{Wt}\left(v_{n-1}\right)+\operatorname{Wt}\left(v_{0}\right)=$ $\mathrm{Wt}\left(v_{n-1}\right)+(1,0)=\mathrm{Wt}\left(v_{n-1}\right)+b$. Assume that $n$ is odd, by Lemma 4.2. $\left[v_{1}, x_{0} v_{n-1}\right]=x_{0} \cdots x_{n-2} v_{n}=w$ and $\operatorname{Wt}(w)=\mathrm{Wt}\left(v_{n-1}\right)+\mathrm{Wt}\left(v_{1}\right)-\mathrm{Wt}\left(v_{0}\right)=\mathrm{Wt}\left(v_{n-1}\right)+(-1,1)=\mathrm{Wt}\left(v_{n-1}\right)+a$. Now (D) is proved.

$\left(\mathrm{A}^{\prime}\right),\left(\mathrm{B}^{\prime}\right)$ Now we combine monomials $\cup_{n \geq 3} T_{n}^{1}$ in one infinite broken line. The united broken line satisfies (A) and $(B)$, respectively. By $(\mathrm{C})$, we know the final part of $T_{n-1}^{1}$ and the initial part of $T_{n}^{1}$. By (D), we insert between them either $b$ or $a$, thus yielding $\left(\mathrm{A}^{\prime}\right)$. By (D), consider a connecting segment $a$ (or $b$ ) between $T_{n-1}^{1}$ and $T_{n}^{1}$, that goes to the next connecting segment between $T_{n}^{1}$ and $T_{n+1}^{1}$ which is $b$ (respectively, $a$ ) followed by the prefix $b$. Thus, we obtain the same replacement rule for the letter of the connecting segment, yielding $\left(\mathrm{B}^{\prime}\right)$.

By drawing, one checks that by $\left(\mathrm{A}^{\prime}\right)$ for any $k \geq 3$ the united broken line $\cup_{n \geq 3} T_{n}^{1}$ contains one or two vertices of degree $k$. It remains to view a few points of $T_{0}^{1} \cup T_{1}^{1} \cup T_{2}^{1}$ on Fig. 2 and see that the second claim is proved.

(F) The local pattern around $v_{n}$ moves to the local pattern around $v_{n+2}, n \geq 3$. This fact follows from (C) and (D).

Now we are ready to prove the first claim. Let $T_{n}^{2}$ be the set of monomials of the second type of length $n, n \geq 2$, which are drawn blue on Fig. 2. By Lemma 8.1, monomials of the second type are obtained from ones of the first type by shifts: $T_{n+1}^{2}=T_{n}^{1}+(-1)^{n}(-2,1), n \geq 3$. Using the first claim, each piece $T_{n+1}^{2}$, $n \geq 1$, contains one or two monomials of fixed degree. A problem is that we cut the united green broken line into pieces and apply different shifts $\pm(-2,1)$ to them, increasing or decreasing the degree. Observe that (F) applies to monomials of two types as well. Around the even pivot elements (like $v_{4}$, $\left.v_{6}\right)$, these shifts are in "opposite directions". Around the odd pivot elements (like $v_{3}, v_{5}$ ), this effect gives three blue points of fixed degree on two lines, but the total number of monomials of fixed degree remains at most 4 (see the pairs of dashed grey lines on Fig. 2). By (F), the same patterns are retained in neighborhoods of the even and odd pivot elements. Collecting green and blue points together we get $\{2,3,4\}$ monomials of fixed degree, thus proving the first claim.

Consider the third claim. By Corollary 5.3. we take all monomials of the first type and add squares of the pivot elements. The squares $v_{n}^{2}=x_{n+1} v_{n+2}, n$ being odd, yield totally three basis monomials on lines passing through them, see the lines passing through $v_{3}^{2}$ and $v_{5}^{2}$ on Fig. 2 ,

Corollary 11.2. Let char $K=2, \mathbf{R}$ the Lie algebra generated by $\left\{v_{0}, v_{1}\right\}$ (i.e. without p-mapping), and $a_{n}=\operatorname{dim} \mathbf{R}_{n}=\operatorname{dim} \mathbf{R}^{n} / \mathbf{R}^{n+1}, n \geq 1$. Then

i) The sequence $\left(a_{n} \mid n \geq 1\right)$, starting with $n \geq 5$, consists of alternating parts of two types: a block 1,1 followed either by 2 ( $a$ diamond) or by a block 2,2,2 (a triplet of diamonds).

ii) The sequence in not eventually periodic.

Proof. By $\left(\mathrm{A}^{\prime}\right), \cup_{n \geq 3} T_{n}^{1}$ is drawn using the alternating parts: $a$ followed by either $b$ or $b^{3}$. Using the replacement rule $\left(\mathrm{B}^{\prime}\right), \cup_{n \geq 4} T_{n}^{1}$ consists of the alternating parts: $b b$ followed by either $a b$ or $(a b)^{3}$. By drawing the respective broken line one observes that the last parts yield one or three diamonds, while the first part yields components 1,1. The initial coefficients are seen on Fig. 2, see also below.

The first part explains the origin of diamonds. The eventual periodicity of the degree components would mean eventual periodicity of the sequence of letters $a, b$. The sequence of monomials contains infinitely many $v_{n}, n \geq 0$, close to the diagonal because $\operatorname{swt}\left(v_{n}\right)= \pm 1$. An eventual periodicity would mean that the monomials of the first type $w$ lie in a strip $|\operatorname{swt}(w)| \leq C$. This is not the case because values $\operatorname{swt}\left(x_{0} x_{2} \cdots x_{2 k-2} v_{2 k}\right)=1-k, k \geq 2$, are not bounded.

Remark 3. We obtain an interesting eventually non-periodic sequence $\left(a_{n} \mid n \geq 1\right)$, which was not found in the database [58. Below, a triplet of diamonds 2,2,2 denote as $2^{3}$ : 
Corollary 11.3. Let char $K=2$ and $\mathbf{R}$ the Lie algebra generated by $\left\{v_{0}, v_{1}\right\}$. Then $\mathbf{R}$ is of width 2 but it is not thin.

Proof. By Picture 2, $\operatorname{dim} \mathbf{R}_{15}=\operatorname{dim} \mathbf{R}_{16}=2$. Let $0 \neq z \in \mathbf{R}_{8,7} \subset \mathbf{R}_{15}$. The covering property (Subsection [1.5) is not satisfied because $\left[\mathbf{R}_{1}, z\right]=\left\langle\left[v_{0}, z\right]+\left[v_{1}, z\right]\right\rangle_{K} \subset \mathbf{R}_{9,7}+\mathbf{R}_{8,8}=\mathbf{R}_{8,8} \varsubsetneqq \mathbf{R}_{7,9} \oplus \mathbf{R}_{8,8}=\mathbf{R}_{16}$.

Remark 4. Since $\mathbf{R}$ above is not thin, the respective diamonds should be called fake diamonds.

\section{Lie SUperalgebra $\mathbf{R}=\mathbf{R}_{\overline{0}} \oplus \mathbf{R}_{\overline{1}}$ Is Nil GRADED, NON-NILLITy of $\mathbf{A}$}

In this section we establish that the Lie superalgebra $\mathbf{R}$ enjoys a property analogous to the periodicity of the Grigorchuk and Gupta-Sidki groups. Namely, we prove that $\mathbf{R}=\mathbf{R}_{\overline{0}} \oplus \mathbf{R}_{\overline{1}}$ is ad-nil $\mathbb{Z}_{2}$-graded (Theorem 12.1). Recall that $\mathbb{Z}^{2}$-components of $\mathbf{R}$ are at most one-dimensional (Theorem 8.4). In particular, this implies that an extension of Martinez-Zelmanov Theorem 1.1 for the Lie superalgebra case in characteristic zero is not possible (Corollary 12.2). We also prove that the components $\mathbf{R}_{ \pm}$and $\mathbf{A}_{ \pm}$of the triangular decompositions of Corollary 6.4 are locally nilpotent (Theorem 12.3 ).

On the other hand, in case char $K=0$ and char $K=2$, we show that the associative algebra $\mathbf{A}=$ $\operatorname{Alg}\left(v_{0}, v_{1}\right)$ is not nil, the restricted Lie algebra $\mathbf{R}=\operatorname{Lie}_{p}\left(v_{0}, v_{1}\right)$, char $K=2$, is also not nil (Lemma 12.4).

Theorem 12.1. Let $\mathbf{R}=\operatorname{Lie}\left(v_{0}, v_{1}\right)=\mathbf{R}_{\overline{0}} \oplus \mathbf{R}_{\overline{1}}$. For any $a \in \mathbf{R}_{\bar{n}}, \bar{n} \in\{\overline{0}, \overline{1}\}$, the operator $\operatorname{ad}(a)$ is nilpotent.

Proof. First, assume that $a \in \mathbf{R}_{\overline{1}}$. We have $(\operatorname{ad} a)^{2}=\operatorname{ad}\left(a^{2}\right)$, so it is sufficient to establish nilpotence of $\operatorname{ad}\left(a^{2}\right)$, where $a^{2}=\frac{1}{2}[a, a] \in \mathbf{R}_{\overline{0}}$ (in case char $K=2$, we use the formal square mapping $\mathbf{R}_{\overline{1}} \ni a \mapsto a^{2} \in \mathbf{R}_{\overline{0}}$ ). Thus, the proof is reduced to the case of an even element $a \in \mathbf{R}_{\overline{0}}$. Observe that even standard monomials contain at least one Grassmann variable, because the pivot elements $v_{n}$ are odd.

We prove a more general statement. Fix a number $N>0$. Let $V$ be a set of standard monomials of length at most $N$, each containing at least one Grassmann letter. We are going to prove that the associative algebra $\operatorname{Alg}(V) \subset$ End $\Lambda$ is nilpotent.

Let $w$ be a nonzero product of $M$ elements $w_{i} \in V$. We get $\operatorname{wt}(w) \geq M$, because $\operatorname{wt}\left(w_{i}\right) \geq 1$. We transform the product as follows. We move to the left and order Grassmann variables, while not changing an order of the heads. Observe that a Grassmann variable $x_{j}$ can either disappear by commuting with an appropriated head $v_{k}, k \leq j$, or be substituted by a product of smaller Grassmann letters. The following examples show the commutation patterns:

$$
\begin{aligned}
& x_{2} v_{5} \cdot x_{5} v_{7}=-x_{2} x_{5} \cdot v_{5} v_{7}+x_{2} \cdot v_{7} \\
& x_{3} v_{4} \cdot x_{5} v_{7}=-x_{3} x_{5} \cdot v_{4} v_{7} \\
& x_{2} v_{3} \cdot x_{5} v_{7}=-x_{2} x_{5} \cdot v_{3} v_{7}+x_{2} x_{3} x_{4} \cdot v_{7} .
\end{aligned}
$$

As a result, the product can be written as a linear combination of monomials of the form

$$
u=x_{i_{1}} \cdots x_{i_{n}} \cdot v_{k_{1}} \cdots v_{k_{m}}, \quad i_{1}<\cdots<i_{n}<N, \quad k_{l} \leq N .
$$

By notations above, consider that $n$ is the number of Grassmann variables and $m$ the number of heads in the product above. Since $x_{i_{l}} \in\left\{x_{0}, \ldots, x_{N-1}\right\}$ and the product $u$ is nonzero, we have $n \leq N$. On the other hand, the number of Grassmann variables in the original product $w$ is greater or equal to the number of the heads, and this property is kept by all transformations (23), hence $m \leq n \leq N$. We evaluate weight of the resulting monomial $u$ :

$$
M \leq \operatorname{wt}(w)=\operatorname{wt}(u) \leq m \cdot \operatorname{wt}\left(v_{N}\right)=m \cdot 2^{N} \leq N \cdot 2^{N} .
$$

Therefore, $\operatorname{Alg}(V)^{N_{1}}=0$, where $N_{1}=N \cdot 2^{N}+1$.

Now, consider any even element $a \in \mathbf{R}_{\overline{0}}$. Then $a$ is a finite linear combination of even standard monomials, which contain at least one Grassmann variable, let $N$ be the maximum of their lengths. By the arguments above, $a^{N_{1}}=0$. Set $k=2 N_{1}-1$. Let $l_{a}, r_{a}$ be operators of left and right multiplications by $a$ in $\mathbf{A}$, respectively. For any $b \in \mathbf{R}$, we have

$$
(\operatorname{ad} a)^{k}(b)=\left(l_{a}-r_{a}\right)^{k}(b)=\sum_{i+j=k}\left(\begin{array}{c}
k \\
i
\end{array}\right)(-1)^{j} l_{a}^{i} r_{a}^{j}(b)=\sum_{i+j=k}\left(\begin{array}{c}
k \\
i
\end{array}\right)(-1)^{j} a^{i} b a^{j}=0 .
$$

Remark 5. We do not know whether ad $a$ is nilpotent where $a \in \mathbf{R}$ is a non- $\mathbb{Z}_{2}$-homogeneous element.

To compare with the case of Lie algebras (Theorem 1.1) we need a weaker version on the $\mathbb{Z}^{2}$-gradation.

Corollary 12.2. Consider the multidegree $\mathbb{Z}^{2}$-gradation $\mathbf{R}=\underset{n_{1}, n_{2} \geq 0}{\oplus} \mathbf{R}_{n_{1} n_{2}}$ (Lemma 6.3). For any $a \in$ $\mathbf{R}_{n_{1} n_{2}}, n_{1}, n_{2} \geq 0$, the operator $\operatorname{ad}(a)$ is nilpotent. 
Proof. One has $\mathbf{R}_{n_{1}, n_{2}} \subset \mathbf{R}_{\overline{n_{1}+n_{2}}}$ for all $n_{1}, n_{2} \geq 0$.

Theorem 12.3. Consider the triangular decompositions of Corollary 6.4.

i) in $\mathbf{A}=\mathbf{A}_{+} \oplus \mathbf{A}_{0} \oplus \mathbf{A}_{-}$, the components $\mathbf{A}_{+}, \mathbf{A}_{-}$are locally nilpotent;

ii) in $\mathbf{R}=\mathbf{R}_{+} \oplus \mathbf{R}_{0} \oplus \mathbf{R}_{-}$, all three components are locally nilpotent.

Proof. The homogeneous components of $\mathbf{R}$ and $\mathbf{A}$ are in regions of plane bounded by pairs of logarithmic curves (Theorem $\mathbf{7 . 4}$ and Theorem 9.3, see also Fig. 1). In case of the positive and negative components, the result easily follows by the geometric arguments of [44, Corollary 5.2].

Consider the subalgebra $\mathbf{R}_{0}$, it is spanned by non-pivot standard monomials, i.e. they contain Grassmann variables. Now the arguments of Theorem 12.1 yield that $\operatorname{Alg}\left(\mathbf{R}_{0}\right)$ is locally nilpotent.

Lemma 12.4. Let char $K=0$ or char $K=2$. Then

i) The associative algebra $\mathbf{A}=\operatorname{Alg}\left(v_{0}, v_{1}\right)$ is not nil.

ii) Let char $K=2$. The restricted Lie algebra $\mathbf{R}=\operatorname{Lie}_{p}\left(v_{0}, v_{1}\right)$ is not nil.

Proof. Let char $K=2$. Recall that the Lie superalgebra $\mathbf{R}=\operatorname{Lie}\left(v_{1}, v_{2}\right)$ coincides with the restricted Lie algebra $\operatorname{Lie}_{p}\left(v_{0}, v_{1}\right)$ generated by $v_{0}, v_{1}$ (Corollary 5.3). So, for any $a \in \mathbf{R}$ one has the square belonging to $\mathbf{R}$. Fix $n \geq 0$ and $0 \neq \alpha \in K$. Consider $v=v_{n}+\alpha x_{n} v_{n+1} \in \mathbf{R}$. We have

$$
\begin{aligned}
v^{2} & =\left(v_{n}+\alpha x_{n} v_{n+1}\right)^{2}=v_{n}^{2}+\left[v_{n}, \alpha x_{n} v_{n+1}\right] \\
& =x_{n+1} v_{n+2}+\alpha v_{n+1}=\alpha\left(v_{n+1}+1 / \alpha \cdot x_{n+1} v_{n+2}\right) ; \\
\left(v^{2}\right)^{2} & =\alpha^{2} \cdot 1 / \alpha\left(v_{n+2}+\alpha x_{n+2} v_{n+3}\right)=\alpha\left(v_{n+2}+\alpha x_{n+2} v_{n+3}\right) .
\end{aligned}
$$

By induction, we get

$$
v^{2^{k}}=\alpha^{r(k)}\left(v_{n+k}+\alpha^{(-1)^{k}} x_{n+k} v_{n+k+1}\right) \neq 0, \quad \text { where } r(k) \in \mathbb{N}, \quad k \geq 0 .
$$

We conclude that $\mathbf{R}$ is not nil as a restricted Lie algebra. Hence, its associative hull $\mathbf{A}$ is not nil as well.

Now, let char $K=0$, and $\mathbb{F}_{2}$ the field with two elements. A $\mathbb{Z}$-span of pure Lie monomials is a subring $\mathbf{W}_{\mathbb{Z}, \text { fin }}\left(\Lambda_{I}\right) \subset \mathbf{W}_{\text {fin }}\left(\Lambda_{I}\right)$ (see notation in Section 6) and infinite sums (5) with coefficients in $\mathbb{Z}$ as well $\mathbf{W}_{\mathbb{Z}}\left(\Lambda_{I}\right) \subset \mathbf{W}\left(\Lambda_{I}\right)$. We use the ordinary square, which is trivial on pure Lie monomials. Reduction modulo 2 yields natural epimorphisms:

$$
\begin{aligned}
\psi: \mathbf{W}_{\mathbb{Z}, \text { fin }}\left(\Lambda_{I}\right) & \rightarrow \mathbf{W}_{\mathbb{Z}, \text { fin }}\left(\Lambda_{I}\right) \otimes_{\mathbb{Z}} \mathbb{F}_{2} \cong \mathbf{W}_{\mathbb{F}_{2}, \text { fin }}\left(\Lambda_{I}\right) ; \\
\psi: \mathbf{W}_{\mathbb{Z}}\left(\Lambda_{I}\right) & \rightarrow \mathbf{W}_{\mathbb{Z}}\left(\Lambda_{I}\right) \otimes_{\mathbb{Z}} \mathbb{F}_{2} \cong \mathbf{W}_{\mathbb{F}_{2}}\left(\Lambda_{I}\right) .
\end{aligned}
$$

Computations of Theorem 5.1 show that we have a $\mathbb{Z}$-subring $\operatorname{Lie}_{\mathbb{Z}}\left(v_{0}, v_{1}\right) \subset \operatorname{Lie}_{K}\left(v_{0}, v_{1}\right)=\mathbf{R} \subset \mathbf{W}(\Lambda)$, consider also $\operatorname{Alg}_{\mathbb{Z}}\left(v_{0}, v_{1}\right) \subset \operatorname{Alg}_{K}\left(v_{0}, v_{1}\right)=\mathbf{A}$. Using Corollary 5.2 and Corollary 5.3 reduction modulo 2 yields epimorphisms of Lie super-rings and associative rings:

$$
\begin{aligned}
& \psi: \operatorname{Lie}_{\mathbb{Z}}\left(v_{0}, v_{1}\right) \rightarrow \operatorname{Lie}_{\mathbb{Z}}\left(v_{0}, v_{1}\right) \otimes_{\mathbb{Z}} \mathbb{F}_{2} \cong \operatorname{Lie}_{\mathbb{F}_{2}}\left(v_{0}, v_{1}\right) ; \\
& \bar{\psi}: \operatorname{Alg}_{\mathbb{Z}}\left(v_{0}, v_{1}\right) \rightarrow \operatorname{Alg}_{\mathbb{Z}}\left(v_{0}, v_{1}\right) \otimes_{\mathbb{Z}} \mathbb{F}_{2} \cong \operatorname{Alg}_{\mathbb{F}_{2}}\left(v_{0}, v_{1}\right) .
\end{aligned}
$$

By the first claim, $\operatorname{Alg}_{\mathbb{F}_{2}}\left(v_{0}, v_{1}\right)$ is not nil, hence $\mathbf{A}$ is not nil as well.

\section{Lie SUPERALGeBRa $\mathbf{R}$ is JUSt Infinite}

A $K$-algebra $A$ is called just infinite dimensional, or simply just infinite, if $\operatorname{dim}_{K} A=\infty$ and each nonzero ideal of $A$ has finite codimension. It is also called hereditary just infinite provided that any ideal of finite codimension is just infinite. Recall that by Lemma 4.3, $\mathbf{R}=\operatorname{Lie}\left(v_{0}, v_{1}\right)$ is infinite dimensional.

Theorem 13.1. The Lie superalgebra $\mathbf{R}=\operatorname{Lie}\left(v_{0}, v_{1}\right)$ is just infinite.

Proof. Assume that $J \triangleleft \mathbf{R}$ is nonzero ideal. Let $0 \neq a \in J$.

A) By Theorem 5.1 (Corollary 5.3 in case char $K=2$ ), $a$ is a finite linear combination of standard monomials. Among these monomials, consider those of greater weight, which are at most two: $w_{1}=r_{N-2} v_{N}$ and $w_{2}=r_{N-2} x_{N} v_{N+1}$ (Lemma 8.1). Thus,

$$
a=\tilde{a}+\alpha r_{N-2} v_{N}+\beta r_{N-2} x_{N} v_{N+1}, \quad \alpha, \beta \in K
$$

where at least one of $\{\alpha, \beta\}$ is nonzero and $\tilde{a}$ contains standard monomials which weights are less than $\operatorname{wt}\left(r_{N-2} v_{N}\right)=\operatorname{wt}\left(r_{N-2} x_{N} v_{N+1}\right)$. We multiply $a$ by all pivot elements $v_{i}$ such that $x_{i}$ is a factor of $r_{N-2}$, 
thus deleting the whole tail $r_{N-2}$. As a result, we get

$$
\begin{gathered}
a^{\prime}=\tilde{a}^{\prime}+\alpha v_{N}+\beta x_{N} v_{N+1} \in J ; \\
1 \leq \operatorname{wt}\left(\tilde{a}^{\prime}\right)<\operatorname{wt}\left(v_{N}\right)=2^{N},
\end{gathered}
$$

where the inequalities above stand for all standard monomials in decomposition of $\tilde{a}^{\prime}$.

B) Assume that $\alpha=0$, then $\beta \neq 0$. In this case we take

$$
a^{\prime \prime}=\left[v_{n}, a^{\prime}\right]=\left[v_{n}, \tilde{a}^{\prime}\right]+\beta v_{N+1} \in J, \quad \beta \neq 0 .
$$

Thus, without loss of generality, we can consider that $\alpha \neq 0$ in (24). Recall that $\left[v_{N-1}^{2}, v_{N}\right]=-v_{N+1}$, $\left[v_{N-1}^{2}, x_{N} v_{N+1}\right]=\left[x_{N} v_{N+1}, x_{N} v_{N+1}\right]=0$ (see Lemma 4.1). Also, we use (24) and estimates (25):

$$
\begin{aligned}
b & =\left[v_{N-1}^{2}, a^{\prime}\right]=b^{\prime}-\alpha v_{N+1} \in J, \quad \text { where } \\
b^{\prime} & =\left[v_{N-1}^{2}, \tilde{a}^{\prime}\right] ; \\
2^{N}<2 \operatorname{wt}\left(v_{N-1}\right)+1 & \leq \operatorname{wt}\left(b^{\prime}\right)<2 \operatorname{wt}\left(v_{N-1}\right)+2^{N}=2^{N+1} .
\end{aligned}
$$

Comparing (27) with bounds of Lemma 7.1. we conclude that $b^{\prime}$ can only have monomials of the first type of length $N+1$ and ones of the second type of length $N+2$. A scalar multiple of (26) has a form:

$$
\tilde{b}=\sum_{i} \lambda_{i} r_{N-1}^{(i)} v_{N+1}+\sum_{j} \mu_{j} \bar{r}_{N-1}^{(j)} x_{N+1} v_{N+2}+v_{N+1} \in J, \quad \lambda_{i}, \mu_{j} \in K .
$$

Since upper bound (27) is strict, all tails $r_{N-1}^{(i)}, \bar{r}_{N-1}^{(j)}$ have at least one Grassmann variable $\left\{x_{0}, \ldots, x_{N-1}\right\}$.

C) Take

$$
\left[x_{0} \cdots x_{N} v_{N+2}, \tilde{b}\right]=-x_{0} \cdots x_{N} x_{N+1} v_{N+3} \in J .
$$

Multiplying by $v_{0}, \ldots, v_{N+1}$, we delete the respective Grassmann letters and obtain $v_{N+3} \in J$.

D) By Lemma 4.1] $\left[v_{N+2}^{2}, v_{N+3}\right]=-v_{N+4} \in J$. Similarly, by induction, $v_{n} \in J$ for all $n \geq N+3$. Let us prove that any standard monomial of length $n \geq N+6$ belongs to $J$. Let $n \geq N+5$. Since $v_{n-2} \in J$ we have

$$
\left[x_{0} \cdots x_{n-3} v_{n-1}, v_{n-2}\right]=-x_{0} \cdots x_{n-2} v_{n} \in J
$$

Multiplying by $v_{i}, i=0, \ldots, n-2$, we can delete any letters $x_{i}$ above. Hence, $J$ contains all standard monomials of the first type of length $n \geq N+5$.

Consider the case char $K \neq 2$. Let $n \geq N+5$ be even. Since $v_{n-2} \in J$, using Lemma 4.2, we have

$$
\left[v_{0}, v_{n-2}\right]=2 x_{0} \cdots x_{n-3} x_{n-1} v_{n} \in J .
$$

Similarly, we can delete any letters $x_{i}, i=0, \ldots, n-3$, above. Let $n \geq N+6$ be odd. Since $v_{n-3} \in J$, using Lemma 4.1, we get

$$
\begin{aligned}
{\left[x_{0} v_{n-4},\left[v_{n-4}, v_{n-3}\right]\right] } & =\left[x_{0} v_{n-4},-x_{n-4} v_{n-2}\right]=-x_{0} v_{n-2} \in J ; \\
{\left[v_{1}, x_{0} v_{n-2}\right] } & =2 x_{0} \cdots x_{n-3} x_{n-1} v_{n} \in J .
\end{aligned}
$$

Similarly, we can delete any letters $x_{i}, i=0, \ldots, n-3$, above. Hence, all standard monomials of the second type of length $n \geq N+6$ belong to $J$.

Consider char $K=2$. Using the formal quadratic mapping, $v_{n-2}^{2}=x_{n-1} v_{n} \in J$ for all $n \geq N+5$.

We proved that $J$ contains all basis monomials of $\mathbf{R}$ of length greater or equal to $N+6$. Therefore, dimension of the quotient algebra $\mathbf{R} / J$ is finite, bounded by number of the basis monomials of $\mathbf{R}$ of length less than $N+6$.

Lemma 13.2. The Lie superalgebra $\mathbf{R}=\operatorname{Lie}\left(v_{0}, v_{1}\right)$ is not hereditary just infinite.

Proof. Fix $m \geq 1$. Let $\mathbf{R}(m) \subset \mathbf{R}$ be the linear span of all basis monomials of $\mathbf{R}$ of length at least $m$. By multiplication rules (see Lemma 4.2 and (9) $), \mathbf{R}(m)$ is an ideal of $\mathbf{R}$. Its codimension is equal to a finite number of the basis monomials of length less than $m$. (In particular, $\operatorname{dim} \mathbf{R} / \mathbf{R}(1)=1)$. Let $J=x_{0} \mathbf{R}(m)$ be the subspace of $\mathbf{R}(m)$ spanned by its basis monomials containing $x_{0}$. By multiplication rules, $J$ is an abelian ideal of $\mathbf{R}(m)$. Since $v_{i} \in \mathbf{R}(m) \backslash J$ for all $i \geq m$, we conclude that $\operatorname{dim} \mathbf{R}(m) / J=\infty$.

Remark 6. We conjecture that the 3-generated Lie superalgebra $\mathbf{Q}$ of [41] is also just infinite but not hereditary just infinite.

Acknowledgments. The authors are grateful to Alexei Krasilnikov, Plamen Koshlukov, Ivan Shestakov, Said Sidki, Dessislava Kochloukova, and Dmitry Millionschikov for useful discussions. 


\section{REFERENCES}

[1] Bahturin Yu. A., Identical relations in Lie algebras. VNU Science Press, Utrecht, 1987.

[2] Bahturin Yu.A., Mikhalev A.A., Petrogradsky V.M., and Zaicev M. V., Infinite dimensional Lie superalgebras, de Gruyter Exp. Math. vol. 7, de Gruyter, Berlin, 1992

[3] Bahturin, Yu.A.; Olshanskii, A., Large restricted Lie algebras, J. Algebra (2007) 310, No. 1, 413-427.

[4] Bahturin, Yu.A.; Sehgal, S.K.; Zaicev, M.V., Group gradings on associative algebras, J. Algebra (2001) 241, No. 2, 677-698.

[5] Bartholdi L., Branch rings, thinned rings, tree enveloping rings. Israel J. Math. 154 (2006), 93-139.

[6] Bartholdi L., Self-similar Lie algebras. J. Eur. Math. Soc. (JEMS) 17 (2015), no. 12, 3113-3151.

[7] Bartholdi L., Grigorchuk R.I., Lie methods in growth of groups and groups of finite width. Computational and geometric aspects of modern algebra,1-27, London Math. Soc. Lecture Note Ser., 275, Cambridge Univ. Press, Cambridge, 2000.

[8] Bouarroudj, S., Grozman, P., Leites, D. Classification of finite dimensional modular Lie superalgebras with indecomposable Cartan matrix, SIGMA 5 (2009), 060, 1-63.

[9] Caranti, A.; Mattarei, S.; Newman, M.F., Scoppola, C.M., Thin groups of prime-power order and thin Lie algebras, Quart. J. Math. Oxford Ser. (2) 47 (187) (1996) 279-296.

[10] Caranti, A.; Mattarei, S.; Newman, M.F. Graded Lie algebras of maximal class. Trans. Am. Math. Soc. 349 (1997) No. 10, 4021-4051.

[11] Caranti, A.; Newman, M.F. Graded Lie algebras of maximal class. II. J. Algebra 229 (2000), No. 2, 750-784.

[12] Drensky V. and Hammoudi L., Combinatorics of words and semigroup algebras which are sums of locally nilpotent subalgebras. Canad. Math. Bull. 47 (2004), no. 3, 343-353.

[13] Elduque, A. Fine gradings on simple classical Lie algebras. J. Algebra 324 (2010), No. 12, 3532-3571.

[14] Ershov M., Golod-Shafarevich groups: a survey, Int. J. Algebra Comput. 22, (2012) No. 5, Article ID 1230001.

[15] Fabrykowski, J., Gupta, N., On groups with sub-exponential growth functions. J. Indian Math. Soc. (N.S.) 49 (1985), no. 3-4, 249-256.

[16] Fialowski A., Classification of graded Lie algebras with two generators, Mosc. Univ. Math. Bull., 38:2 (1983), 76-79.

[17] Golod, E.S. On nil-algebras and finitely approximable p-groups. Am. Math. Soc., Translat., II. Ser. 48, $103-106$ (1965); translation from Izv. Akad. Nauk SSSR, Ser. Mat. 28, 273-276 (1964).

[18] Golod E.S. and Shafarevich I.R., On the class field tower, Am. Math. Soc., Translat., II. Ser. 48, 91-102 (1965); translation from Izv. Akad. Nauk SSSR Ser. Mat. 28 (1964), 261-272;

[19] Golod, E.S. On some problems of Burnside type. Am. Math. Soc., Translat., II. Ser. 84, (1969) 83-88; translation from Tr. Mezdunarod. Kongr. Mat., Moskva 1966, 284-289 (1968).

[20] Grigorchuk, R.I., On the Burnside problem for periodic groups., Funktsional. Anal. i Prilozhen. 14 (1980), no. 1, 53-54.

[21] Grigorchuk, R.I. Degrees of growth of finitely generated groups, and the theory of invariant means. Math. USSR, Izv. 25 (1985), 259-300; translation from Izv. Akad. Nauk SSSR, Ser. Mat. 48 (1984), No.5, 939-985.

[22] Grigorchuk, R.I., Just infinite branch groups. New horizons in pro- $p$ groups, 121-179, Progr. Math., 184, Birkhauser Boston, Boston, MA, 2000.

[23] Gupta N., and Sidki S., On the Burnside problem for periodic groups., Math. Z. 182 (1983), no. 3, 385-388.

[24] Gupta, N.; Sidki, S. Some infinite p-groups. Algebra Logic 22, (1983) 421-424; translation from: Algebra Logika 22 (1983) No. $5,584-589$.

[25] Iohara, K.; Mathieu, O., Classification of simple Lie algebras on a lattice. Proc. Lond. Math. Soc. (3) 106 (2013), no. 3, $508-564$.

[26] Jacobson N., Lie algebras, Interscience, New York. 1962.

[27] Kac, V.G. Lie superalgebras. Adv. Math. 26, (1977), 8-96.

[28] Kelarev A.V., A sum of two locally nilpotent rings may be not nil. Arch. Math. 60 (1993), no. 5, 431-435.

[29] Krause G.R. and Lenagan T.H., Growth of algebras and Gelfand-Kirillov dimension, AMS, Providence, R.I., 2000.

[30] Krylyouk Ia., The enveloping algebra of the Petrogradsky-Shestakov-Zelmanov algebra is not graded-nil in the critical characteristics, J. Lie Theory, 21, (2011), No. 3, 703-709.

[31] Lenagan, T.H., Smoktunowicz Agata., An infinite dimensional affine nil algebra with finite Gelfand-Kirillov dimension, $J$. Am. Math. Soc. 20, (2007) No. 4, 989-1001.

[32] Martinez C., Zelmanov E., Nil algebras and unipotent groups of finite width. Adv. Math. 147, (1999) No.2, 328-344.

[33] Mikhalev A.A., Subalgebras of free Lie p-superalgebras. Math. Notes 43, (1988) No.2, 99-106; translation from Mat. Zametki 43 (1988) No.2, 178-191.

[34] Millionschikov D.V., Graded filiform Lie algebras and symplectic nilmanifolds, in: Geometry, Topology, and Mathematical Physics. 212 American Mathematical Society Translations, Series 2. (2004) 259-279.

[35] Millionschikov D.V., Naturally graded Lie algebras (Carnot algebras) of slow growth. arXiv:1705.07494.

[36] Nekrashevych, V., Self-similar groups. Mathematical Surveys and Monographs 117. Providence, RI: American Mathematical Society (AMS) (2005).

[37] Passman D.S. and Petrogradsky V.M., Polycyclic restricted Lie algebras, Comm. Algebra, 29 (2001), no. 9, 3829-3838.

[38] Petrogradsky V.M., Identities in the enveloping algebras for modular Lie superalgebras. J. Algebra 145 (1992), no. 1, 1-21.

[39] Petrogradsky V.M., On Lie algebras with nonintegral q-dimensions. Proc. Amer. Math. Soc. 125 (1997), no. 3, $649-656$.

[40] Petrogradsky V.M., Examples of self-iterating Lie algebras, J. Algebra, 302 (2006), no. 2, 881-886.

[41] Petrogradsky V., Fractal nil graded Lie superalgebras, J. Algebra, 466 (2016), 229-283.

[42] Petrogradsky V., Nil Lie p-algebras of slow growth, Comm. Algebra. 45, (2017), no. 7, 2912-2941.

[43] Petrogradsky V. M., Yu. P. Razmyslov, and E. O. Shishkin, Wreath products and Kaluzhnin-Krasner embedding for Lie algebras, Proc. Amer. Math. Soc., 135, (2007), 625-636.

[44] Petrogradsky V.M. and Shestakov I.P. Examples of self-iterating Lie algebras, 2, J. Lie Theory, 19 (2009), no. 4, 697-724.

[45] Petrogradsky V.M. and Shestakov I.P. Self-similar associative algebras, J. Algebra, 390 (2013), 100-125.

[46] Petrogradsky V.M. and Shestakov I.P. On properties of Fibonacci restricted Lie algebra, J. Lie Theory, 23 (2013), no. 2, 407-431. 
[47] Petrogradsky V. and Shestakov I.P., Fractal nil graded Lie, associative, Poisson, and Jordan superalgebras, preprint.

[48] Petrogradsky V.M., Shestakov I.P., and Zelmanov E., Nil graded self-similar algebras, Groups Geom. Dyn., 4 (2010), no. 4, $873-900$.

[49] Radford D. E., Divided power structures on Hopf algebras and embedding Lie algebras into special-derivation algebras, $J$. Algebra, 98 (1986), 143-170.

[50] Yu.P. Razmyslov, Identities of algebras and their representations. AMS, Providence RI 1994.

[51] Rozhkov, A.V. Lower central series of a group of tree automorphisms, Math. Notes 60, No.2, 165-174 (1996); translation from Mat. Zametki 60, No.2, 225-237 (1996).

[52] Shestakov I.P. and Zelmanov E., Some examples of nil Lie algebras. J. Eur. Math. Soc. (JEMS) 10 (2008), no. 2, 391-398.

[53] Scheunert, M. The theory of Lie superalgebras. Lecture Notes in Mathematics. 716. Berlin. Springer-Verlag. 1979.

[54] Shalev, A.; Zelmanov, E.I. Narrow Lie algebras: a coclass theory and a characterization of the Witt algebra, J. Algebra 189 (1997) No. 2, 294-331.

[55] Shalev, A.; Zelmanov, E.I. Narrow algebras and groups, J. Math. Sci. (N. Y.) 93 (1999) No. 6, 951-963.

[56] Sidki S.N., Functionally recursive rings of matrices — Two examples. J.Algebra 322 (2009), no. 12, 4408-4429.

[57] Sidki S.N., A primitive ring associated to a Burnside 3-group. J. London Math. Soc. (2) 55 (1997), no. 1, 55-64.

[58] Sloane N.J.A., editor, The On-Line Encyclopedia of Integer Sequences, published electronically at https://oeis.org.

[59] Strade H., Simple Lie algebras over fields of positive characteristic. I: Structure theory. Berlin: de Gruyter. 2004.

[60] Strade H. and Farnsteiner R., Modular Lie algebras and their representations, New York etc.: Marcel Dekker, 1988.

[61] Vergne, M., Cohomologie des algèbres de Lie nilpotentes. Application à l'étude de la variété des algèbres de Lie nilpotentes. Bull. Soc. Math. Fr. 98, (1970). 81-116.

[62] Voden T., Subalgebras of Golod-Shafarevich algebras, Int. J. Algebra Comput. 19, (2009) No. 3, 423-442.

[63] Zelmanov E., Some open problems in the theory of infinite dimensional algebras., J. Korean Math. Soc. 44 (2007), no. 5, $1185-1195$.

Federal Institute of Rio Grande do Norte, 59112-490 Natal RN, Brazil

E-mail address: ottoaugustomorais@gmail.com

Department of Mathematics, University of Brasilia, 70910-900 Brasilia DF, Brazil

E-mail address: petrogradsky@rambler.ru 